\title{
Exotic vectorlike quark phenomenology in the minimal linear $\sigma$ model
}

\author{
J. A. Aguilar-Saavedra, ${ }^{1, *, \|}$ J. Alonso-González@ ${ }^{1,2, \dagger}$ L. Merlo, ${ }^{1,2, \$}$ and J. M. No ${ }^{1,2, \S}$ \\ ${ }^{1}$ Instituto de Física Teórica UAM/CSIC, Calle Nicolás Cabrera 13-15, \\ Cantoblanco E-28049 Madrid, Spain \\ ${ }^{2}$ Departamento de Física Teórica, Universidad Autónoma de Madrid, \\ Cantoblanco E-28049 Madrid, Spain
}

(Received 16 December 2019; accepted 22 January 2020; published 12 February 2020)

\begin{abstract}
Extensions of the Standard Model that include vectorlike quarks commonly also include additional particles that may mediate new production or decay modes. Using the minimal linear $\sigma$ model as an example, which reduces to the minimal $S O(5) / S O(4)$ composite Higgs model in a specific limit, we consider the phenomenology of vectorlike quarks when a scalar singlet $\sigma$ is present. This new particle may be produced in the decays $T \rightarrow t \sigma, B \rightarrow b \sigma$, where $T$ and $B$ are vectorlike quarks of charges $2 / 3$ and $-1 / 3$, respectively, with the subsequent decay $\sigma \rightarrow W^{+} W^{-}, Z Z, h h$. By scanning over the allowed parameter space we find that these decays may be dominant. In addition, we find that the presence of several new particles allows for single $T$ production cross sections larger than those expected in minimal models. We discuss the observability of these new signatures in existing searches.
\end{abstract}

DOI: $10.1103 /$ PhysRevD.101.035015

\section{INTRODUCTION}

The electroweak (EW) hierarchy problem remains one of the weaknesses of the Standard Model (SM) of particle physics. Indeed, a potentially strong fine-tuning in the scalar sector is required once the need for heavy new physics is considered, for example to explain nonvanishing neutrino masses and the presence of dark matter. Among the bestknown scenarios to solve this problem, the composite Higgs $(\mathrm{CH})$ framework [1-3] has received a renewed interest [4-7]. The traditional $\mathrm{CH}$ model is based on a global symmetry $\mathcal{G}$, broken spontaneously to a subgroup $\mathcal{H}$, such that the coset $\mathcal{G} / \mathcal{H}$ is symmetric. A certain number of Nambu-Goldstone bosons (GBs) arise from this breaking, in particular, the SM GBs, which are the would-be longitudinal components of the EW gauge bosons, and the Higgs itself. This technically solves the hierarchy problem, providing symmetry protection for the Higgs mass.

The idea is that an undetermined strong dynamics, acting at a high scale, generates exotic fermionic bound states that

\footnotetext{
*jaas@ugr.es

j.alonso.gonzalez@csic.es

tuca.merlo@uam.es

§josemiguel.no@uam.es

On leave from Universidad de Granada, E-18071 Granada, Spain.
}

Published by the American Physical Society under the terms of the Creative Commons Attribution 4.0 International license. Further distribution of this work must maintain attribution to the author(s) and the published article's title, journal citation, and DOI. Funded by SCOAP ${ }^{3}$. spontaneously break the group $\mathcal{G}$. Moreover, the gauging of the SM symmetries, together with nonuniversal fermion masses, provide an explicit breaking of $\mathcal{G}$ such that a small mass is generated for the Higgs. SM fermion masses are generated by means of the fermion partial compositeness mechanism [8-10], which consists in introducing into the spectrum a series of exotic (typically vectorlike) fermions which act as partners of the SM fermions, with a role similar to that of right-handed (RH) Majorana neutrinos in the type-I seesaw mechanism [11-13] for neutrino masses. In the mass basis, this results in light SM fermions and heavier exotic counterparts. The top quark is the heaviest among the light fermions and it turns out to be largely composed of exotic states. On the other hand, the lightest exotic fermions are the counterparts of the top and bottom quarks. These are labeled, depending on their electric charge, as the top partner $T$ and bottom partner $B$, respectively. The search for heavy partners of the SM quarks is a very active field from the experimental side [14-30]. The discovery of such particles would not only represent a fundamental step toward the understanding of the theory beyond the SM, but also a window to study the electroweak symmetry breaking sector.

In this paper the focus is on the so-called minimal linear $\sigma$ model (ML $\sigma \mathrm{M}$ ) [31], which attempts to improve the limitations of the traditional $\mathrm{CH}$ models. In the latter, the indetermination of the strong dynamics leads to describing the model Lagrangian with an effective approach, which however leads to a limited range of application. The ML $\sigma \mathrm{M}$, instead, is a renormalizable model based on the global spontaneous symmetry breaking $S O(5) \rightarrow S O(4)$ 
that matches the minimal $\mathrm{CH}$ model [4,32-34] in a specific limit. The main idea is the introduction of a scalar quintuplet of $S O(5)$ that contains as degrees of freedom the three SM GBs, the Higgs and an additional EW singlet $\sigma$. Moreover, the spectrum is enlarged by the introduction of several exotic fermions in the trivial and fundamental representations of $S O(5)$ that will give rise to the fermion partial compositeness mechanism.

Several studies have been conducted on this model to analyze its features at low energies [35], projecting to the so-called Higgs effective field theory Lagrangian [36-49], to access its ability to solve the strong $C P$ problem [50-52]. The focus of this paper, instead, is to study the phenomenology associated with the exotic vectorlike quarks (VLQs). Indeed, being a renormalizable model, all the parameters describing the low-energy theory are fixed in terms of the original Lagrangian parameters. In particular, the whole spectrum of the exotic VLQs and their interactions are fixed in terms of the initial parameters: this is a novelty with respect to previous studies where no direct link was present between the masses of the different VLQs or with their interactions, which were taken to be independent parameters.

The new VLQ states can be produced in pairs via QCD interactions in hadron collisions, with cross sections that only depend on the VLQ mass. In addition, it is also possible to singly produce the VLQs at colliders via their mixing with SM quarks. In both cases, these cross sections quickly decrease as the VLQ mass increases, and therefore for collider phenomenology the most interesting signals are generically those of the lightest VLQ. ${ }^{1}$ The electric charge of the lightest VLQ (and therefore its decay modes) depends on the model parameters. In case it has exotic charge $5 / 3$ or $-4 / 3$, its phenomenology is quite the same as in minimal models [53,54], because it does not couple to the scalar $\sigma$. On the other hand, if it has charge $2 / 3(T)$ or charge $-1 / 3(B)$, these VLQ states inherit new decay modes beyond the standard decays

$$
\begin{aligned}
& T \rightarrow W b, \quad T \rightarrow Z t, \quad T \rightarrow h t, \\
& B \rightarrow W t, \quad B \rightarrow Z b, \quad B \rightarrow h b,
\end{aligned}
$$

due to the presence of the singlet scalar $\sigma$. These new decays

$$
T \rightarrow \sigma t, \quad B \rightarrow \sigma b
$$

with

$$
\sigma \rightarrow W^{+} W^{-}, Z Z, h h
$$

\footnotetext{
${ }^{1}$ Since single VLQ production also depends on the fermion mixing, the highest production cross section may not always correspond to the lightest VLQ.
}

open a plethora of new possible signatures for VLQs, both in the pair and single production channels. Current searches, while targeting the standard decays in Eq. (1), are sensitive to the new decays to a varying degree.

Nonstandard VLQ top and bottom partner decays into a singlet scalar field like those in Eq. (2) have already been explored in the literature [55-60] (see also Refs. [61,62]). These studies mainly considered the singlet scalar $\sigma$ as being invisible at colliders $[55,58,63]$, or decaying into $Z \gamma$, $\gamma \gamma$ final states [60], yet they did not focus on the singlet scalar decays from Eq. (3), which generically are the main decay modes for a singlet scalar $\sigma$ mixing with the SM Higgs boson and with mass $m_{\sigma}>200 \mathrm{GeV}$. Moreover, the singlet scalar $\sigma$ has previously been considered to be a GB of the coset $\mathcal{G} / \mathcal{H}$ (together with the Higgs boson), while in the ML $\sigma \mathrm{M}$ the nature of the $\sigma$ field is qualitatively very different from that of the Higgs (e.g., $\sigma$ is naively expected to be significantly heavier than the Higgs boson).

The paper is organized as follows. Section II describes the relevant aspects of the model, where we write down the interaction Lagrangians that determine the phenomenology of the VLQs. The constraints on the parameter space of the model arising either from precision electroweak data, Higgs boson measurements, or direct searches, are discussed in Sec. III. In Sec. IV we outline the procedure used for the exploration of the ML $\sigma \mathrm{M}$ parameter space. The results of our parameter scan are analyzed in Sec. V, focusing on the phenomenology of the lightest VLQ, the presence of new decay modes and opportunities for single VLQ production at the LHC. We also include a discussion on how these new decay modes can be experimentally searched for. We finally conclude in Sec. VI.

\section{THE MINIMAL LINEAR $\sigma$ MODEL}

The ML $\sigma \mathrm{M}$ is based on the group $S O(5) \times U(1)_{X}$, where the last factor ensures the correct hypercharge assignments for the SM fields. The spectrum contains the four SM gauge bosons associated to the SM gauge symmetry, a real scalar field $\phi$ in the fundamental representation of $S O(5)$, the elementary fermions with the same quantum numbers as in the SM and finally exotic vectorlike quarks in the trivial and fundamental representations of $S O(5)$.

The scalar field $\phi$ includes the three would-be longitudinal components of the SM gauge bosons $\pi_{i}, i=1,2,3$, the Higgs field $h$ and the additional scalar field $s$, which is a singlet under the SM group:

$$
\phi=\left(\pi_{1}, \pi_{2}, \pi_{3}, \mathrm{~h}, \mathrm{~s}\right)^{T} \stackrel{\text { u.g. }}{\longrightarrow} \phi=(0,0,0, \mathrm{~h}, \mathrm{~s})^{T},
$$

where the last expression holds in the unitary gauge (but in the unbroken phase). The associated scalar potential is responsible for the spontaneous $S O(5) \rightarrow S O(4)$ breaking. As discussed in the following, explicit breaking terms are 
TABLE I. Transformation properties of the fields in the spectrum under $S O(5) \times U(1)_{X}$. The superscripts $(2 / 3)$ and $(-1 / 3)$ on the fermionic fields refer to the top and bottom quark sectors.

\begin{tabular}{lccccc}
\hline \hline & $\phi$ & $\psi^{(2 / 3)}$ & $\chi^{(2 / 3)}$ & $\psi^{(-1 / 3)}$ & $\chi^{(-1 / 3)}$ \\
\hline$S O(5)$ & 5 & 5 & 1 & 5 & 1 \\
$U(1)_{X}$ & 0 & $+2 / 3$ & $+2 / 3$ & $-1 / 3$ & $-1 / 3$ \\
\hline \hline
\end{tabular}

also contained in the scalar potential and are responsible for the $S O(4)$ and EW breaking.

The elementary fermions do not directly couple to the scalar $\phi$, and therefore neither does the SM Higgs once $S O(5)$ is broken. These couplings only arise through the mediation of the exotic fermions that do have tree-level interactions with $\phi$. The proto-Yukawas are couplings between the $S O(5)$ quintuplet VLQs $\psi$ and the singlets $\chi$ : these fields have $U(1)_{X}$ charge equal to $2 / 3(-1 / 3)$ and are associated to the up-type (down-type) sector.

Table I reports the fields in the spectrum together with their transformation properties under $S O(5) \times U(1)_{X}$. While in full generality one set of VLQs can be introduced per SM generation, in this paper only the third-generation SM fermions and their VLQ siblings are considered, accordingly to the description of the original publication [31]. This does not represent a restriction in the present analysis, because the top and bottom partners are indeed the lightest VLQs and therefore they are the first exotic states that may show up in experiments, as discussed before. Moreover, their contributions are the only numerically relevant ones and for this reason the tree-level couplings of the first two generation quarks are taken to be purely SM like (deviations from the SM can however be induced at loop level and will be discussed in Sec. III).

In the remainder of this section, the scalar and fermionic sectors will be discussed following Ref. [31], fixing the notation and the conventions that will be used in the rest of the paper.

\section{A. The scalar sector}

The part of the Lagrangian describing the interactions of the scalar fields and the symmetry breaking is

$$
\mathscr{L}_{\phi}=\frac{1}{2}\left(D_{\mu} \phi\right)^{T}\left(D^{\mu} \phi\right)-V(\phi)
$$

where the covariant derivative containing the $S U(2)_{L} \times$ $U(1)_{Y}$ gauge bosons is defined by

$$
D_{\mu} \phi=\left(\partial_{\mu}+i g \Sigma_{L}^{a} W_{\mu}^{a}+i g^{\prime} \Sigma_{R}^{3} B_{\mu}\right) \phi,
$$

where $\Sigma_{L}^{a}$ and $\Sigma_{R}^{a}$ denote the generators of $S U(2)_{L} \times$ $S U(2)_{R}$, which is isomorphic to $S O(4)^{\prime}$, a subgroup of $S O(5)$, and rotated with respect to the $S O(4)$ residual group after the spontaneous breaking of $S O(5)$. The scalar potential $V(\phi)$ contains terms responsible for the spontaneous breaking of $S O(5)$ down to $S O(4)$, plus additional terms that explicitly break the residual $S O(4)$,

$$
V(\phi)=\lambda\left(\phi^{T} \phi-f^{2}\right)^{2}+\alpha f^{3} \mathrm{~s}-\beta f^{2} \mathrm{~h}^{2},
$$

where $f$ is the scale at which the $S O(5)$ breaking takes place. The last two terms are just a subset of all the soft breaking terms, but they are the ones necessary to absorb divergences once the one-loop Coleman-Weinberg contributions are considered (see Ref. [51] for a different treatment). For values $\lambda \gg 1$, the nonlinear model would be recovered, corresponding to a decoupling of the $\sigma$ field.

The $S O(4)$ and EW breaking then require $\mathrm{h}$ and $\mathrm{s}$ to develop a vacuum expectation value (VEV)

$$
\langle\mathrm{h}\rangle=v_{\mathrm{h}}, \quad\langle\mathrm{s}\rangle=v_{\mathrm{s}},
$$

where the normalization has been chosen to match Eq. (4) (note in particular that $v_{\mathrm{h}}=246 \mathrm{GeV}$ ). For $\alpha \neq 0 \neq \beta$, the VEVs turn out to be

$$
v_{\mathrm{s}}^{2}=f^{2} \frac{\alpha^{2}}{4 \beta^{2}}, \quad v_{\mathrm{h}}^{2}=f^{2}\left(1-\frac{\alpha^{2}}{4 \beta^{2}}+\frac{\beta}{2 \lambda}\right),
$$

which satisfy the condition

$$
v_{\mathrm{h}}^{2}+v_{\mathrm{s}}^{2}=f^{2}\left(1+\frac{\beta}{2 \lambda}\right) .
$$

Requiring the $S O(5)$ breaking $\left(f^{2}>0\right)$ and that the Higgs arises as a GB $\left(\left|v_{\mathrm{h}}\right|<\left|v_{\mathrm{s}}\right|\right)$ imply that

$$
2 \beta^{2}\left(1+\frac{\beta}{2 \lambda}\right)<\alpha^{2}<4 \beta^{2}\left(1+\frac{\beta}{2 \lambda}\right)
$$

which in the strongly interacting limit, $\alpha, \beta \ll \lambda$, reduces to

$$
2 \beta^{2} \leq \alpha^{2} \leq 4 \beta^{2} .
$$

Then, in order to get $v_{\mathrm{h}}^{2} \ll f^{2}$ from Eq. (9), $\alpha / 2 \beta \sim 1$ is needed.

Once the scalar fields develop their VEVs, a nondiagonal $2 \times 2$ mass matrix results from Eq. (7). Diagonalizing this mass matrix, the mass eigenstates $h, \sigma$ turn out to be

$$
h=\mathrm{h} \cos \gamma-\mathrm{s} \sin \gamma, \quad \sigma=\mathrm{h} \sin \gamma+\mathrm{s} \cos \gamma,
$$

where the mixing angle is given by

$$
\tan 2 \gamma=\frac{4 v_{\mathrm{h}} v_{\mathrm{s}}}{3 v_{\mathrm{s}}^{2}-v_{\mathrm{h}}^{2}-f^{2}},
$$

and the masses of these two states, in the limit of $\alpha, \beta \ll \lambda$ and positive $\beta$, read

$$
m_{\sigma}^{2} \simeq 8 \lambda f^{2}+2 \beta\left(3 f^{2}-v_{\mathrm{h}}^{2}\right), \quad m_{h}^{2} \simeq 2 \beta v_{\mathrm{h}}^{2} .
$$


As a concluding remark, the possibility $m_{\sigma}<m_{h}$ is viable but extremely fine-tuned and therefore will not be considered. As a consequence, the angle $\gamma$ will always be taken in the interval $[-\pi / 4, \pi / 4]$.

In the interaction basis, only the SM GBs and $h$ couple to the EW gauge bosons. Due to the rotation in Eq. (13), $\sigma$ inherits couplings with $W^{ \pm}$and $Z$ weighted by $\sin \gamma$, while the $h$ couplings acquire a suppression with $\cos \gamma$,

$$
\begin{aligned}
\mathscr{L}_{\phi} \supset & -\lambda\left(h^{2}+\sigma^{2}+2 h \sigma\right)^{2}+ \\
& -4 \lambda\left(v_{\mathrm{h}} \cos \gamma-v_{\mathrm{S}} \sin \gamma\right)\left(h^{3}+h \sigma^{2}\right)+ \\
& -4 \lambda\left(v_{\mathrm{h}} \sin \gamma+v_{\mathrm{S}} \cos \gamma\right)\left(\sigma^{3}+\sigma h^{2}\right) \\
& +\left(1+\frac{h}{v_{\mathrm{h}}} \cos \gamma+\frac{\sigma}{v_{\mathrm{h}}} \sin \gamma\right) \\
& \times\left(M_{W}^{2} W_{\mu}^{+} W^{\mu-}+\frac{1}{2} M_{Z}^{2} Z_{\mu} Z^{\mu}\right) .
\end{aligned}
$$

The modification in the $h$ couplings with respect to SM predictions constrain the possible values of $\gamma$, as will be seen in Sec. III.
It is then straightforward to obtain the partial widths to SM bosons of both the $h$ and $\sigma$ :

$$
\begin{aligned}
\Gamma\left(h \rightarrow W W^{*}\right) & =\Gamma_{\mathrm{SM}}\left(h \rightarrow W W^{*}\right) \cos ^{2} \gamma, \\
\Gamma\left(h \rightarrow Z Z^{*}\right) & =\Gamma_{\mathrm{SM}}\left(h \rightarrow Z Z^{*}\right) \cos ^{2} \gamma, \\
\Gamma\left(\sigma \rightarrow W^{+} W^{-}\right) & =\frac{\sqrt{2} G_{F}}{16 \pi} m_{\sigma}^{3} \sin ^{2} \gamma\left[1+\mathcal{O}\left(\frac{M_{W}^{2}}{m_{\sigma}^{2}}\right)\right], \\
\Gamma(\sigma \rightarrow Z Z) & =\frac{\sqrt{2} G_{F}}{32 \pi} m_{\sigma}^{3} \sin ^{2} \gamma\left[1+\mathcal{O}\left(\frac{M_{Z}^{2}}{m_{\sigma}^{2}}\right)\right], \\
\Gamma(\sigma \rightarrow h h) & =\frac{\sqrt{2} G_{F}}{32 \pi} m_{\sigma}^{3} \sin ^{2} \gamma\left[1+\mathcal{O}\left(\frac{M_{h}^{2}}{m_{\sigma}^{2}}\right)\right] .
\end{aligned}
$$

\section{B. The fermionic sector}

We now turn to discuss the fermionic sector of the $\mathrm{ML} \sigma \mathrm{M}$. In the basis of canonical kinetic terms for the gauge fields, the renormalizable fermionic Lagrangian can be written as follows [31]:

$$
\begin{aligned}
\mathscr{L}_{f}= & \overline{\mathrm{q}}_{L} i \not \mathrm{d}_{L}+\overline{\mathrm{t}}_{R} i D \mathrm{t}_{R}+\bar{b}_{R} i D D \mathrm{~b}_{R} \\
& +\bar{\psi}^{(2 / 3)}\left[i \not D-M_{5}\right] \psi^{(2 / 3)}+\bar{\chi}^{(2 / 3)}\left[i \not D-M_{1}\right] \chi^{(2 / 3)}+\bar{\psi}^{(-1 / 3)}\left[i \not D-M_{5}^{\prime}\right] \psi^{(-1 / 3)}+\bar{\chi}^{(-1 / 3)}\left[i \not D-M_{1}^{\prime}\right] \chi^{(-1 / 3)} \\
& -\left[\mathrm{y}_{1} \bar{\psi}_{L}^{(2 / 3)} \phi \chi_{R}^{(2 / 3)}+\mathrm{y}_{2} \bar{\psi}_{R}^{(2 / 3)} \phi \chi_{L}^{(2 / 3)}+\mathrm{y}_{1}^{\prime} \bar{\psi}_{L}^{(-1 / 3)} \phi \chi_{R}^{(-1 / 3)}+\mathrm{y}_{2}^{\prime} \bar{\psi}_{R}^{(-1 / 3)} \phi \chi_{L}^{(-1 / 3)}\right. \\
& +\Lambda_{1}\left(\bar{q}_{L} \Delta_{2 \times 5}^{(2 / 3)} \psi_{R}^{(2 / 3)}\right)+\Lambda_{2} \bar{\psi}_{L}^{(2 / 3)}\left(\Delta_{5 \times 1}^{(2 / 3)} \mathrm{t}_{R}\right)+\Lambda_{3} \bar{\chi}_{L}^{(2 / 3)} \mathrm{t}_{R}+\Lambda_{1}^{\prime}\left(\overline{\mathrm{q}}_{L} \Delta_{2 \times 5}^{(-1 / 3)} \psi_{R}^{(-1 / 3)}\right) \\
& \left.+\Lambda_{2}^{\prime} \bar{\psi}_{L}^{(-1 / 3)}\left(\Delta_{5 \times 1}^{(-1 / 3)} \mathrm{b}_{R}\right)+\Lambda_{3}^{\prime} \bar{\chi}_{L}^{(-1 / 3)} \mathrm{b}_{R}+\text { H.c. }\right],
\end{aligned}
$$

where the $U(1)_{X}$ charge of the exotic fermion fields $\psi$ and $\chi$ has been made explicit throughout. The first line contains the canonical kinetic terms for the elementary quarks: $q_{L}$ for the left-handed (LH) $S U(2)_{L}$ doublet, $t_{R}$ and $\mathrm{b}_{R}$ for the RH $S U(2)_{L}$ singlets. The second line describes the kinetic and mass terms for the exotic quarks. The proto-Yukawa interactions among the exotic fermions and the scalar quintuplet are written in the third line. Finally the last two lines show the $S O(5)$-breaking interactions between the exotic and elementary quarks: the terms $\Delta_{2 \times 5}$ and $\Delta_{5 \times 1}$ denote spurion fields [64-68] that connect the exotic and elementary sectors and are responsible for the light fermion masses. According to the fermion partial compositeness paradigm, no direct elementary fermion couplings to $\phi$ are allowed. All the parameters in the previous Lagrangian are taken to be real: this is equivalent to assuming $C P$ conservation in these interactions; as only the third generation of light fermions are considered and therefore the CabibboKobayashi-Maskawa matrix cannot be described, this hypothesis is viable.
In terms of the $S U(2)_{L}$ quantum numbers, the scalar quintuplet $\phi$ and the VLQs can be decomposed as follows:

$$
\begin{aligned}
\phi & =\left(H^{T}, \tilde{H}^{T}, \mathrm{~s}\right), & \\
\psi^{(2 / 3)} & \sim\left(\mathrm{K}, \mathrm{Q}, \mathrm{T}_{5}\right)^{T}, & \chi^{(2 / 3)} \sim \mathrm{T}_{1}, \\
\psi^{(-1 / 3)} & \sim\left(\mathrm{Q}^{\prime}, \mathrm{K}^{\prime}, \mathrm{B}_{5}\right)^{T}, & \chi^{(-1 / 3)} \sim \mathrm{B}_{1},
\end{aligned}
$$

where $H$ is the $\mathrm{SM} S U(2)_{L}$ doublet, with $\tilde{H} \equiv i \sigma_{2} H^{*}$, and $\mathrm{K}^{(\prime)}$ and $\mathrm{Q}^{(\prime)}$ are $S U(2)_{L}$ doublets and $\mathrm{T}_{1,5}$ and $\mathrm{B}_{1,5}$ are singlets. The rest of the charge assignments can be seen in Table II, where the hypercharge follows from the relation

$$
Y=\Sigma_{R}^{(3)}+X,
$$

where $X$ is the $U(1)_{X}$ charge and $\Sigma_{R}^{(3)}$ is the third component of the global $S U(2)_{R}$, which is part of the residual $S O(4)$ group after the breaking of $S O(5)$.

In terms of the $S U(2)_{L}$ components, the fermionic Lagrangian acquires the following form: 
TABLE II. Decompositions of the exotic fields and their transformations under the SM group.

\begin{tabular}{lcccccc}
\hline \hline Charge/Field & $\mathrm{K}$ & $\mathrm{Q}$ & $\mathrm{T}_{1,5}$ & $\mathrm{Q}$ & $\mathrm{Q}^{\prime}$ & $\mathrm{K}_{1,5}$ \\
\hline$\Sigma_{R}^{(3)}$ & $+1 / 2$ & $-1 / 2$ & 0 & $+1 / 2$ & $-1 / 2$ & 0 \\
$S U(2)_{L} \times U(1)_{Y}$ & $(2,+7 / 6)$ & $(2,+1 / 6)$ & $(1,+2 / 3)$ & $(2,+1 / 6)$ & $(2,-5 / 6)$ & $(1,-1 / 3)$ \\
$U(1)_{X}$ & $+2 / 3$ & $+2 / 3$ & $+2 / 3$ & $-1 / 3$ & $-1 / 3$ & $-1 / 3$ \\
$U(1)_{E M}$ & $\mathrm{~K}^{u}=+5 / 3$ & $Q^{u}=+2 / 3$ & & $\mathrm{Q}^{\prime u}=+2 / 3$ & $\mathrm{~K}^{\prime u}=-1 / 3$ & \\
& $\mathrm{~K}^{d}=+2 / 3$ & $\mathrm{Q}^{d}=-1 / 3$ & $+2 / 3$ & $\mathrm{Q}^{\prime d}=-1 / 3$ & $\mathrm{~K}^{\prime d}=-4 / 3$ & $-1 / 3$ \\
\hline \hline
\end{tabular}

$$
\begin{aligned}
& \mathscr{L}_{f}=\overline{\mathrm{q}}_{L} i D \mathrm{q}_{L}+\overline{\mathrm{\epsilon}}_{R} i D \mathrm{t}_{R}+\overline{\mathrm{b}}_{R} i \not D \mathrm{~b}_{R} \\
& +\overline{\mathrm{K}}\left[i \not D-M_{5}\right] \mathrm{K}+\overline{\mathrm{Q}}\left[i \not D-M_{5}\right] \mathrm{Q}+\overline{\mathrm{T}}_{5}\left[i \not D-M_{5}\right] \mathrm{T}_{5}+\overline{\mathrm{T}}_{1}\left[i \not D-M_{1}\right] \mathrm{T}_{1} \\
& +\overline{\mathrm{Q}}^{\prime}\left[i \not D-M_{5}^{\prime}\right] \mathrm{Q}^{\prime}+\overline{\mathrm{K}}^{\prime}\left[i \not D-M_{5}^{\prime}\right] \mathrm{K}^{\prime}+\overline{\mathrm{B}}_{5}\left[i \not D-M_{5}^{\prime}\right] \mathrm{B}_{5}+\overline{\mathrm{B}}_{1}\left[i \not D-M_{1}^{\prime}\right] \mathrm{B}_{1} \\
& -\left[\mathrm{y}_{1}\left(\overline{\mathrm{K}}_{L} H \mathrm{~T}_{1, R}+\overline{\mathrm{Q}}_{L} \tilde{H} \mathrm{~T}_{1, R}+\overline{\mathrm{T}}_{5, L} \mathrm{ST}_{1, R}\right)+\mathrm{y}_{2}\left(\overline{\mathrm{T}}_{1, L} H^{\dagger} \mathrm{K}_{R}+\overline{\mathrm{T}}_{1, L} \tilde{H}^{\dagger} \mathrm{Q}_{R}+\overline{\mathrm{T}}_{1, L} \mathrm{ST}_{5, R}\right)\right. \\
& +\mathrm{y}_{1}^{\prime}\left(\overline{\mathrm{Q}}_{L}^{\prime} H \mathrm{~B}_{1, R}+\overline{\mathrm{K}}_{L}^{\prime} \tilde{H}_{1, R}+\overline{\mathrm{B}}_{5, L} \mathrm{sB}_{1, R}\right)+\mathrm{y}_{2}^{\prime}\left(\overline{\mathrm{B}}_{1, L} H^{\dagger} \mathrm{Q}_{R}^{\prime}+\overline{\mathrm{B}}_{1, L} \tilde{H}^{\dagger} \mathrm{K}_{R}^{\prime}+\overline{\mathrm{B}}_{1, L} \mathrm{sB}_{5, R}\right) \\
& +\Lambda_{1} \overline{\mathrm{q}}_{L} \mathrm{Q}_{R}+\Lambda_{2} \overline{\mathrm{T}}_{5, L} \mathrm{t}_{R}+\Lambda_{3} \overline{\mathrm{T}}_{1, L} \mathrm{t}_{R}+\Lambda_{1}^{\prime} \overline{\mathrm{q}}_{L} \mathrm{Q}_{R}^{\prime}+\Lambda_{2}^{\prime} \overline{\mathrm{B}}_{5, L} \mathrm{~b}_{R}+\Lambda_{3}^{\prime} \overline{\mathrm{B}}_{1, L} \mathrm{~b}_{R}+\text { H.c.], }
\end{aligned}
$$

where the scalar fields $H$ and $s$ still denote the unshifted and unrotated fields defined in Eq. (4). In order to match with the notation adopted in the previous section, notice that in the unitary gauge

$$
H \equiv\left(\begin{array}{c}
0 \\
\mathrm{~h} / \sqrt{2}
\end{array}\right)
$$

Light fermion masses arise via a series of interactions à $l a$ the seesaw mechanism and once the Higgs doublets develop a VEV. The diagram in Fig. 1 exemplifies the topquark case. A first-order approximation for the values of the top and bottom quark masses is given by

$$
m_{t} \approx y_{1} \frac{\Lambda_{1} \Lambda_{3}}{M_{1} M_{5}} \frac{v_{\mathrm{h}}}{\sqrt{2}}, \quad m_{b} \approx y_{1}^{\prime} \frac{\Lambda_{1}^{\prime} \Lambda_{3}^{\prime}}{M_{1}^{\prime} M_{5}^{\prime}} \frac{v_{\mathrm{h}}}{\sqrt{2}} .
$$

A more precise result can be obtained by diagonalizing the whole fermion mass matrix that includes elementary and exotic quarks. It is useful to group all the fermions within a single vector,

$$
\Psi=\left(\mathrm{K}^{u}, \mathcal{T}, \mathcal{B}, \mathrm{K}^{\prime}\right)^{T},
$$

where the ordering of the components is based on their electric charges, $+5 / 3,+2 / 3,-1 / 3$, and $-4 / 3$, respectively. Moreover, $\mathcal{T}$ and $\mathcal{B}$ list together all the states with the same electric charge, $+2 / 3$ and $-1 / 3$, respectively,

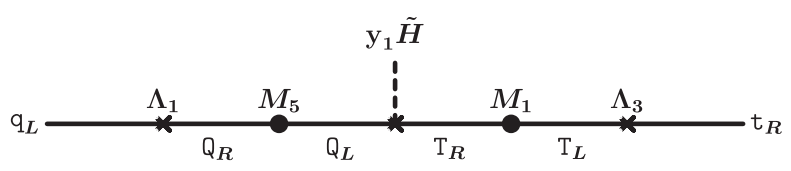

FIG. 1. Top-quark mass term diagram.

$$
\begin{aligned}
& \mathcal{T}=\left(\mathrm{t}, \mathrm{Q}^{u}, \mathrm{~K}^{d}, \mathrm{~T}_{5}, \mathrm{~T}_{1}, \mathrm{Q}^{\prime u}\right)^{T}, \\
& \mathcal{B}=\left(\mathrm{b}, \mathrm{Q}^{\prime d}, \mathrm{~K}^{u}, \mathrm{~B}_{5}, \mathrm{~B}_{1}, \mathrm{Q}^{d}\right)^{T} .
\end{aligned}
$$

The whole fermion mass term, still in the interaction basis, can then be written as

$$
\mathscr{L}_{\mathcal{M}}=-\bar{\Psi}_{L} \mathcal{M}\left(v_{\mathrm{h}}, v_{\mathrm{s}}\right) \Psi_{R},
$$

where the mass matrix $\mathcal{M}\left(v_{\mathrm{h}}, v_{\mathrm{s}}\right)$ is a $14 \times 14$ block diagonal matrix,

$\mathcal{M}\left(v_{\mathrm{h}}, v_{\mathrm{s}}\right)=\operatorname{diag}\left(M_{5}, \mathcal{M}_{\mathcal{T}}\left(v_{\mathrm{h}}, v_{\mathrm{s}}\right), \mathcal{M}_{\mathcal{B}}\left(v_{\mathrm{h}}, v_{\mathrm{s}}\right), M_{5}^{\prime}\right)$,

with

$$
\mathcal{M}_{\mathcal{T}}\left(v_{\mathrm{h}}, v_{\mathrm{s}}\right)=\left(\begin{array}{cccccc}
0 & \Lambda_{1} & 0 & 0 & 0 & \Lambda_{1}^{\prime} \\
0 & M_{5} & 0 & 0 & \mathrm{y}_{1} \frac{v_{\mathrm{h}}}{\sqrt{2}} & 0 \\
0 & 0 & M_{5} & 0 & \mathrm{y}_{1} \frac{v_{\mathrm{h}}}{\sqrt{2}} & 0 \\
\Lambda_{2} & 0 & 0 & M_{5} & \mathrm{y}_{1} v_{\mathrm{S}} & 0 \\
\Lambda_{3} & \mathrm{y}_{2} \frac{v_{\mathrm{h}}}{\sqrt{2}} & \mathrm{y}_{2} \frac{v_{\mathrm{h}}}{\sqrt{2}} & \mathrm{y}_{2} v_{\mathrm{S}} & M_{1} & 0 \\
0 & 0 & 0 & 0 & 0 & M_{5}^{\prime}
\end{array}\right),
$$

and similarly for $\mathcal{M}_{\mathcal{B}}\left(v_{\mathrm{h}}, v_{\mathrm{s}}\right)$, replacing the unprimed parameters with the primed ones and vice versa. The matrix in Eq. (27) can be diagonalized through a biunitary transformation,

$$
\hat{\Psi}_{L, R}=U_{L, R} \Psi \Rightarrow \hat{\mathcal{M}}=U_{L} \mathcal{M} U_{R}^{\dagger},
$$


where $\hat{\Psi}_{L, R}$ stand for the mass eigenstates and $\widehat{\mathcal{M}}$ stands for the diagonal matrix. The two unitary matrices can be written as block-diagonal structures

$$
U_{L, R}=\operatorname{diag}\left(1, U_{L, R}^{\mathcal{T}}, U_{L, R}^{\mathcal{B}}, 1\right),
$$

where $U_{L, R}^{\mathcal{T}}$ and $U_{L, R}^{\mathcal{B}}$ diagonalize $\mathcal{M}_{\mathcal{T}}\left(v_{\mathrm{h}}, v_{\mathrm{S}}\right)$ and $\mathcal{M}_{\mathcal{B}}\left(v_{\mathrm{h}}, v_{\mathrm{s}}\right)$, respectively. Finally, the diagonalized mass matrix is given by

$$
\hat{\mathcal{M}}=\operatorname{diag}\left(M_{5}, \hat{\mathcal{M}}_{\mathcal{T}}, \hat{\mathcal{M}}_{\mathcal{B}}, M_{5}^{\prime}\right)
$$

and the mass eigenstate fermion fields are defined as

$$
\begin{aligned}
& \hat{\Psi}=\left(K^{u}, \hat{\mathcal{T}}, \hat{\mathcal{B}}, K^{\prime d}\right)^{T}, \\
& \hat{\mathcal{T}}=\left(t, T, T_{2}, T_{3}, T_{4}, T_{5}\right)^{T}, \\
& \hat{\mathcal{B}}=\left(b, B, B_{2}, B_{3}, B_{4}, B_{5}\right)^{T},
\end{aligned}
$$

with both charge $2 / 3$ and charge $-1 / 3$ mass eigenstates ordered by increasing mass, so that the lightest states correspond to the top and bottom quarks, respectively. The exotic charge states do not mix with the other fields and then $K^{u} \equiv \mathrm{K}^{u}$ and $K^{\prime d} \equiv \mathrm{K}^{\prime d}$. The full expression for the tree-level top quark mass is given by

$$
\begin{aligned}
m_{t}= & \frac{\mathrm{y}_{1} \Lambda_{1} \Lambda_{3} v_{\mathrm{h}} / \sqrt{2}}{M_{1} M_{5}-\mathrm{y}_{1} \mathrm{y}_{2}\left(v_{h}^{2}+v_{\sigma}^{2}\right)}+ \\
& -\frac{\mathrm{y}_{1} \mathrm{y}_{2} \Lambda_{1} \Lambda_{2} v_{\mathrm{s}} v_{\mathrm{h}} / \sqrt{2}}{M_{1} M_{5}^{2}-\mathrm{y}_{1} \mathrm{y}_{2} M_{5}\left(v_{h}^{2}+v_{\sigma}^{2}\right)},
\end{aligned}
$$

and similarly for the bottom quark mass, replacing the unprimed parameters with the primed ones. This expression matches the one in Eq. (23) once the contributions proportional to $y_{2}$ and $\Lambda_{2}$ are neglected.

\section{Interactions with the $\mathrm{EW}$ gauge bosons}

A large part of the phenomenological discussion in the next sections will follow from the fermion interactions with the EW gauge bosons, which are modified with respect to the SM case due to the mixing between the elementary and exotic quarks. This is the case for the $Z b \bar{b}$ coupling, the electroweak precision observables (EWPOs) and the single production of the VLQs and their decays.

Adopting a compact notation, the Lagrangian terms describing these interactions with the $W^{ \pm}$and $Z$ gauge bosons read

$$
\begin{aligned}
& \mathscr{L}_{W}=\frac{g}{\sqrt{2}} \overline{\hat{\Psi}} \gamma^{\mu}\left(V_{L} P_{L}+V_{R} P_{R}\right) \hat{\Psi} W_{\mu}^{+}+\text {H.c., } \\
& \mathscr{L}_{Z}=\frac{g}{2 c_{W}} \overline{\hat{\Psi}} \gamma^{\mu}\left(C_{L} P_{L}+C_{R} P_{R}\right) \hat{\Psi} Z_{\mu},
\end{aligned}
$$

where $\hat{\Psi}$ are the mass eigenstates in Eq. (32), while the matrices $V$ and $C$ are obtained by means of the unitary matrices $U_{L, R}$ in Eq. (30) and the matrices containing the fermion interactions in the interaction basis. Notice that $V_{L, R}$ are block off-diagonal and $C_{L, R}$ are block diagonal because of charge conservation. $P_{R, L}=\left(1 \pm \gamma_{5}\right) / 2$ are the usual chirality projectors, $g$ is the $S U(2)_{L}$ gauge coupling and $c_{W} \equiv \cos \theta_{W}$, where $\theta_{W}$ is the weak mixing angle. For $\mathscr{L}_{W}$, the coupling matrices in this basis read

$$
V_{L, R}=U_{L, R} \mathcal{V}_{L, R} U_{L, R}^{\dagger},
$$

where $\mathcal{V}_{L, R}$ are the $W$-coupling matrices in the interaction basis, whose form can be deduced from Table II,

$$
\mathcal{V}_{L, R}=\left(\begin{array}{cccc}
0 & \mathcal{V}^{K^{u}} \mathcal{T} & 0_{1 \times 6} & 0 \\
0_{6 \times 1} & 0_{6 \times 6} & \mathcal{V}_{L, R}^{\mathcal{T}} & 0_{6 \times 1} \\
0_{6 \times 1} & 0_{6 \times 6} & 0_{6 \times 6} & \mathcal{V}^{\mathcal{B} K^{\prime d}} \\
0 & 0_{1 \times 6} & 0_{1 \times 6} & 0
\end{array}\right),
$$

where

$$
\begin{aligned}
\mathcal{V}^{K^{u} \mathcal{T}}=\left(\mathcal{V}^{\mathcal{B} K^{\prime d}}\right)^{\dagger}=(0,0,1,0,0,0), \\
\mathcal{V}_{L}^{\mathcal{T} \mathcal{B}}=\left(\begin{array}{lllllll}
1 & 0 & 0 & 0 & 0 & 0 \\
0 & 0 & 0 & 0 & 0 & 1 \\
0 & 0 & 0 & 0 & 0 & 0 \\
0 & 0 & 0 & 0 & 0 & 0 \\
0 & 0 & 0 & 0 & 0 & 0 \\
0 & 1 & 0 & 0 & 0 & 0
\end{array}\right),
\end{aligned}
$$

and $\mathcal{V}_{R}^{\mathcal{T} \mathcal{B}}$ is identical to $\mathcal{V}_{L}^{\mathcal{T} B}$, except for its $(1,1)$ entry which is vanishing,

$$
\begin{aligned}
\left(\mathcal{V}_{R}^{\mathcal{T} B}\right)_{i j} & =\left(\mathcal{V}_{L}^{\mathcal{T} \mathcal{B}}\right)_{i j} \quad \forall(i, j) \neq(1,1), \\
\left(\mathcal{V}_{R}^{\mathcal{T} \mathcal{B}}\right)_{1,1} & =0,
\end{aligned}
$$

corresponding to the fact that right-handed weak eigenstates are $S U(2)_{L}$ singlets.

For $\mathscr{L}_{Z}$, the coupling matrices for mass eigenstates are given by

$$
C_{L, R}=U_{L, R} \mathcal{C}_{L, R} U_{L, R}^{\dagger}-2 s_{W}^{2} \mathcal{Q},
$$

where $s_{W} \equiv \sin \theta_{W}, \mathcal{Q}$ is the electromagnetic charge matrix

$$
\mathcal{Q}=\operatorname{diag}\left(\frac{5}{3}, \frac{2}{3} \mathbb{1}_{6 \times 6},-\frac{1}{3} \mathbb{1}_{6 \times 6},-\frac{4}{3}\right),
$$

and $\mathcal{C}_{L, R}$ is the isospin-dependent coupling matrices in the interaction basis 


$$
\begin{aligned}
\mathcal{C}_{L, R} & =\operatorname{diag}\left(1, \mathcal{C}_{L, R}^{\mathcal{T}}, \mathcal{C}_{L, R}^{\mathcal{B}}, 1\right), \\
\mathcal{C}_{L}^{\mathcal{T}} & =-\mathcal{C}_{L}^{\mathcal{B}}=\operatorname{diag}(1,1,-1,0,0,1), \\
\mathcal{C}_{R}^{\mathcal{T}} & =-\mathcal{C}_{R}^{\mathcal{B}}=\operatorname{diag}(0,1,-1,0,0,1) .
\end{aligned}
$$

The normalization adopted is such that the diagonal entries equal two times the isospin of the weak eigenstates.

\section{Interactions with the scalars}

The Lagrangian terms involving the fermion couplings to the scalar fields are

$$
\mathcal{L}_{s}=\overline{\hat{\Psi}}_{L} \mathrm{Y}_{h} \hat{\Psi}_{R} h+\overline{\hat{\Psi}}_{L} \mathrm{Y}_{\sigma} \hat{\Psi}_{R} \sigma+\text { H.c. }
$$

where $\mathrm{Y}_{h}$ and $\mathrm{Y}_{\sigma}$ are the respective Yukawa coupling matrices of the scalar mass eigenstates, given by

$$
\begin{array}{ll}
\mathrm{Y}_{h}=U_{L} \mathscr{Y}_{h} U_{R}^{\dagger}, & \mathscr{Y}_{h}=\operatorname{diag}\left(0, \mathscr{Y}_{h}^{\mathcal{T}}, \mathscr{Y}_{h}^{\mathcal{B}}, 0\right), \\
\mathrm{Y}_{\sigma}=U_{L} \mathscr{Y}_{\sigma} U_{R}^{\dagger}, & \mathscr{Y}_{\sigma}=\operatorname{diag}\left(0, \mathscr{Y}_{\sigma}^{\mathcal{T}}, \mathscr{Y}_{\sigma}^{\mathcal{B}}, 0\right),
\end{array}
$$

where

$$
\begin{aligned}
& \mathscr{Y}_{h}^{\mathcal{T}}=\left(\begin{array}{cccccc}
0 & 0 & 0 & 0 & 0 & 0 \\
0 & 0 & 0 & 0 & -\mathrm{y}_{1} \frac{c_{\gamma}}{\sqrt{2}} & 0 \\
0 & 0 & 0 & 0 & -\mathrm{y}_{1} \frac{c_{\gamma}}{\sqrt{2}} & 0 \\
0 & 0 & 0 & 0 & \mathrm{y}_{1} s_{\gamma} & 0 \\
0 & -\mathrm{y}_{2} \frac{c_{\gamma}}{\sqrt{2}} & -\mathrm{y}_{2} \frac{c_{\gamma}}{\sqrt{2}} & \mathrm{y}_{2} s_{\gamma} & 0 & 0 \\
0 & 0 & 0 & 0 & 0 & 0
\end{array}\right), \\
& \mathcal{Y}_{h}^{\mathcal{B}}=\mathscr{Y}_{h}^{\mathcal{T}}\left(\mathrm{y}_{i} \rightarrow \mathrm{y}_{i}^{\prime}\right), \\
& \mathcal{Y}_{\sigma}^{\mathcal{T}}=\mathscr{Y}_{h}^{\mathcal{T}}\left(c_{\gamma} \rightarrow s_{\gamma}, s_{\gamma} \rightarrow-c_{\gamma}\right), \\
& \mathscr{Y}_{\sigma}^{\mathcal{B}}=\mathcal{Y}_{\sigma}^{\mathcal{T}}\left(\mathrm{y}_{i} \rightarrow \mathrm{y}_{i}^{\prime}\right),
\end{aligned}
$$

with $c_{\gamma}$ and $s_{\gamma}$ being the cosine and sine of the mixing angle $\gamma$, respectively.

\section{PARAMETER SPACE CONSTRAINTS}

As described in the previous section, the presence of the additional scalar singlet $\sigma$ and the exotic fermions modifies the interactions of the light fermions with respect to the SM. This section is dedicated to summarizing the theoretical and experimental constraints on the parameter space of the ML $\sigma \mathrm{M}$. A few theoretical constraints in the parameter space have already been listed in Sec. II A. First of all, $f^{2}>0$ in order to guarantee that $S O(5) \rightarrow S O(4)$ breaking takes place. Moreover, $\alpha$ and $\beta$ should satisfy the condition in Eq. (11). Finally, by the diagonalization of the scalar mass matrix, $\beta>0$ allows a positive mass for the physical $h$ as shown in Eq. (15).

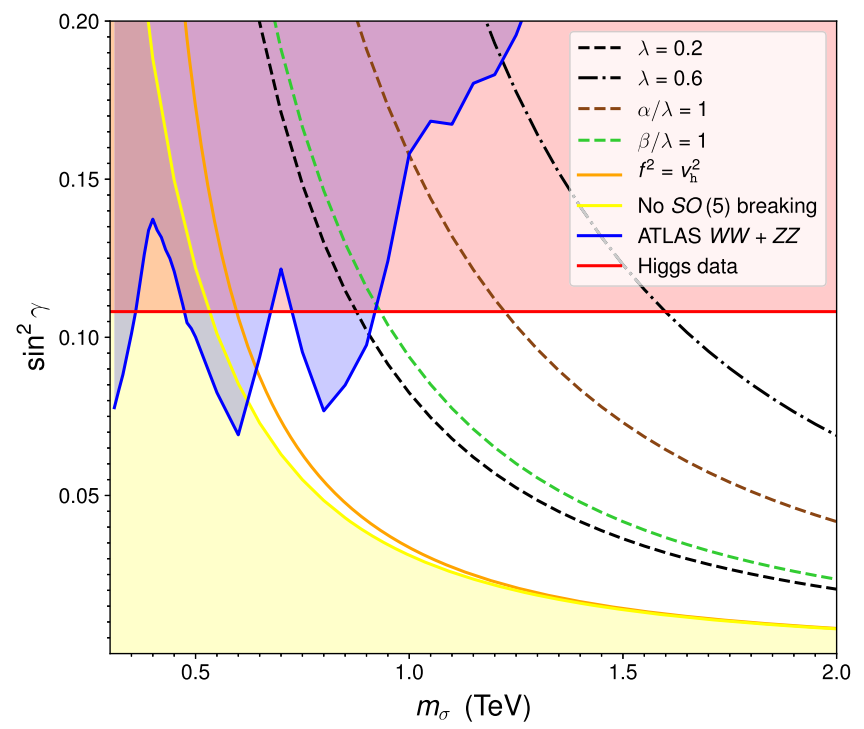

FIG. 2. Constraints on the mass $\left(m_{\sigma}\right)$ and mixing angle $\left(\sin ^{2} \gamma\right)$ of the new singlet scalar $\sigma$ (see text for details).

\section{A. Bounds from LHC Higgs searches}

The measurements of the $125 \mathrm{GeV}$ Higgs signal strengths at the LHC by ATLAS and CMS constrain the Higgs-singlet mixing $\sin \gamma$, which universally suppresses the couplings of $h$ to SM particles with respect to their SM values. Very recently, ATLAS performed a $\sqrt{s}=13 \mathrm{TeV}$ analysis of Higgs signal strengths with $80 \mathrm{fb}^{-1}$ of integrated luminosity [69], from which we derive the bound

$$
\sin ^{2} \gamma \lesssim 0.11
$$

at 95\% C.L. using a $\chi^{2}$ fit to the ATLAS data by assuming a universal suppression of Higgs couplings. This bound is shown in Fig. 2 as an excluded shaded red region in the $\left(m_{\sigma}, \sin ^{2} \gamma\right)$ plane. In addition, Fig. 2 highlights the impact of theoretical constraints on the ML $\sigma \mathrm{M}$ parameter space: the area under the yellow curve is ruled out when requiring a feasible spontaneous symmetry breaking of the ML $\sigma \mathrm{M}$ $S O(5)$ group. Note that realizations where $v_{\mathrm{h}}^{2} / f^{2} \equiv \xi=1$ (where $\xi$ is the nonlinearity parameter typically introduced in $\mathrm{CH}$ models), depicted by the orange line, and even regions where $v_{\mathrm{h}}^{2}>f^{2}$ are not excluded by experimental bounds. Finally, the bound from Eq. (45) is consistent with existing bounds on $\xi$ in the nonlinear limit $m_{\sigma} \gg m_{h}$, for which $\xi \simeq \sin ^{2} \gamma$ [31]. For completeness, Fig. 2 shows other lines where the $S O(5)$-preserving parameter $\lambda$ takes particular values and where it is equal to the remaining parameters of the scalar potential of Eq. (7).

Additional constraints on the ML $\sigma \mathrm{M}$ parameter space arise from ATLAS and CMS searches for heavy scalars decaying into SM gauge boson pairs, $W W$ and $Z Z$. In order to derive the corresponding constraints from these searches, it is useful to introduce an effective Higgs-singlet mixing 
angle $\sin ^{2} \gamma_{\text {eff }}$ as the ratio between the cross sections for gluon-fusion production of $\sigma$ in the $\mathrm{ML} \sigma \mathrm{M}$ (taking into account the VLQ loop contributions to $g g \rightarrow \sigma$, whose expressions can be found in Ref. [31]) and for gluon-fusion production of a SM-like scalar $h_{m_{\sigma}}$, with mass $m_{\sigma}$

$$
\sin ^{2} \gamma_{\mathrm{eff}}=\frac{\sigma(g g \rightarrow \sigma)_{\mathrm{ML} \sigma \mathrm{M}}}{\sigma\left(g g \rightarrow h_{m_{\sigma}}\right)_{\mathrm{SM}}} .
$$

In the absence of VLQ loop contributions, one simply has $\sin ^{2} \gamma_{\text {eff }}=\sin ^{2} \gamma$. Considering the latest $\sqrt{s}=13 \mathrm{TeV}$ ATLAS search for scalar resonances in diboson final states with $36 \mathrm{fb}^{-1}$ of integrated luminosity [70], Fig. 2 shows the 95\% C.L. limits in $\sin ^{2} \gamma_{\text {eff }}$ as an excluded blue area, in the absence of VLQ loop contributions. These latter contributions of VLQs to $g g \rightarrow \sigma$ are instead included when performing the $\mathrm{ML} \sigma \mathrm{M}$ viable parameter space scan in Sec. IV. For the case $y_{2}=y_{2}^{\prime}=0$, these corrections turn out to be negligible, and $\sin ^{2} \gamma_{\text {eff }} \simeq \sin ^{2} \gamma$. On the other hand, for $\mathrm{y}_{2} \in[3.0,6.0]$ (see below) the VLQ loop contributions are important and may interfere constructively with the SM top quark contribution, significantly enhancing the $g g \rightarrow \sigma$ production cross section. Numerically the excluded region in the $\left(m_{\sigma}, \sin ^{2} \gamma_{\text {eff }}\right)$ plane corresponds to the blue region in Fig. 2, replacing $\gamma$ by $\gamma_{\text {eff }}$, up to a small difference due to the variation of the branching ratios of $\sigma \rightarrow t \bar{t}$.

\section{B. Bounds from $Z \boldsymbol{b} \bar{b}$ coupling}

The couplings of the $Z$ boson to the $c$ and $b$ quarks have been precisely measured at LEP. The tree-level mixing of the bottom quark with the VLQs induces deviations from the SM prediction of these couplings (at tree level for the bottom quark and at loop level for the charm quark). The effective $Z b \bar{b}$ vertex can be written as [54]

$$
\mathscr{L}_{Z b b}=-\frac{g}{2 c_{W}} \bar{b} \gamma^{\mu}\left(c_{L} P_{L}+c_{R} P_{R}\right) b Z_{\mu} .
$$

The coefficients can be written as the sum of the SM prediction and its deviation,

$$
c_{L, R}=c_{L, R}^{\mathrm{SM}}+\delta c_{L, R},
$$

where the SM values are given by

$$
c_{L}^{\mathrm{SM}}=1-\frac{2}{3} s_{W}^{2}, \quad c_{R}^{\mathrm{SM}}=-\frac{2}{3} s_{W}^{2} .
$$

Comparing Eqs. (34) and (47), the deviations $\delta c_{L, R}$ read

$$
\delta c_{L, R}=-\left(U_{L, R}^{\mathcal{B}} \mathcal{C}_{L, R}^{\mathcal{B}}\left(U_{L, R}^{\mathcal{B}}\right)^{\dagger}\right)_{1,1}-k_{L, R},
$$

where $k_{L}=1$ and $k_{R}=0$.
The effects of deviations from the SM values can be seen in several observables, such as the ratios of partial widths $R_{b} \equiv \Gamma(b \bar{b}) / \Gamma$ (hadrons) and $R_{c} \equiv \Gamma(c \bar{c}) /$ $\Gamma$ (hadrons), the forward-backward (FB) charge asymmetry $A_{\mathrm{FB}}^{b}$, and the coupling parameter $A_{b}$ from the LR FB asymmetry $[54,71]$ :

$$
\begin{aligned}
R_{b} & =R_{b}^{\mathrm{SM}}\left(1-1.820 \delta c_{L}+0.336 \delta c_{R}\right), \\
A_{\mathrm{FB}}^{b} & =A_{\mathrm{FB}}^{b \mathrm{SM}}\left(1-0.1640 \delta c_{L}-0.8877 \delta c_{R}\right), \\
A_{b} & =A_{b}^{\mathrm{SM}}\left(1-0.1640 \delta c_{L}-0.8877 \delta c_{R}\right) \\
R_{c} & =R_{c}^{\mathrm{SM}}\left(1+0.500 \delta c_{L}-0.0924 \delta c_{R}\right) .
\end{aligned}
$$

The SM values read

$$
\begin{aligned}
R_{b}^{\mathrm{SM}} & =0.21582, \\
A_{\mathrm{FB}}^{b, \mathrm{SM}} & =0.1030, \\
A_{b}^{\mathrm{SM}} & =0.9347, \\
R_{c}^{\mathrm{SM}} & =0.17221,
\end{aligned}
$$

while the experimental results that will be used to constrain the parameter space are [72]

$$
\begin{aligned}
R_{b}^{\exp } & =0.21629 \pm 0.00066, \\
A_{\mathrm{FB}}^{b, \exp } & =0.0992 \pm 0.0016, \\
A_{b}^{\exp } & =0.923 \pm 0.020, \\
R_{c}^{\exp } & =0.1721 \pm 0.003,
\end{aligned}
$$

and, with same ordering as in Eq. (53), the correlation matrix is given by

$$
\rho=\left(\begin{array}{cccc}
1 & -0.10 & -0.08 & -0.18 \\
-0.10 & 1 & 0.06 & 0.04 \\
-0.08 & 0.06 & 1 & 0.04 \\
-0.18 & 0.04 & 0.04 & 1
\end{array}\right)
$$

\section{Bounds from EWPOs}

The EWPOs also set constraints on the parameter space. In our analysis we restrict ourselves to the $T$ and $S$ oblique parameters, which are the most relevant ones. Due to the mixing in the scalar sector, the physical $\sigma$ acquires couplings with the EW gauge bosons, weighted by $\sin \gamma$, and therefore it contributes to the oblique parameters. On the other side, the $h$ couplings get suppressed by $\cos \gamma$ with respect to the SM and this also affects the contributions to $T$ and $S$. All in all, the $T$ and $S$ contributions can be straightforwardly derived from the SM one: 


$$
\begin{aligned}
& T_{\mathrm{ML} \sigma \mathrm{M}}^{h}=c_{\gamma}^{2} T_{\mathrm{SM}}^{h}, \\
& T_{\mathrm{ML} \sigma \mathrm{M}}^{\sigma}=s_{\gamma}^{2} T_{\mathrm{SM}}^{h}\left(m_{h} \rightarrow m_{\sigma}\right), \\
& S_{\mathrm{ML} \sigma \mathrm{M}}^{h}=c_{\gamma}^{2} S_{\mathrm{SM}}^{h}, \\
& S_{\mathrm{ML} \sigma \mathrm{M}}^{\sigma}=s_{\gamma}^{2} S_{\mathrm{SM}}^{h}\left(m_{h} \rightarrow m_{\sigma}\right),
\end{aligned}
$$

so that

$$
\begin{aligned}
\Delta T^{(h, \sigma)} & \equiv T_{\mathrm{ML} \sigma \mathrm{M}}^{(h, \sigma)}-T_{\mathrm{SM}}^{h} \\
& =s_{\gamma}^{2}\left[-T_{\mathrm{SM}}^{h}+T_{\mathrm{SM}}^{h}\left(m_{h} \rightarrow m_{\sigma}\right)\right], \\
\Delta S^{(h, \sigma)} & \equiv S_{\mathrm{ML} \sigma \mathrm{M}}^{(h, \sigma)}-S_{\mathrm{SM}}^{h} \\
& =s_{\gamma}^{2}\left[-S_{\mathrm{SM}}^{h}+S_{\mathrm{SM}}^{h}\left(m_{h} \rightarrow m_{\sigma}\right)\right] .
\end{aligned}
$$

The general expressions for the $T, S$ parameters considering contributions from VLQs with arbitrary couplings can be found in Refs. [73,74], and are given in the Appendix A for completeness. In the SM scenario, that is considering only the SM top and bottom quarks with their EW couplings, the contribution to the $T$ parameter is

$$
\begin{aligned}
T_{\mathrm{SM}}^{f}= & \frac{3}{16 \pi s_{W}^{2} c_{W}^{2} m_{Z}^{2}} \\
& \times\left[m_{t}^{2}+m_{b}^{2}-2 \frac{m_{t}^{2} m_{b}^{2}}{m_{t}^{2}-m_{b}^{2}} \log \left(\frac{m_{t}^{2}}{m_{b}^{2}}\right)\right]
\end{aligned}
$$$$
\approx 1.19 \text {. }
$$

It is then possible to define the deviation from the SM contribution as the difference

$$
\Delta T^{f}=T_{\mathrm{ML} \sigma \mathrm{M}}^{f}-T_{\mathrm{SM}}^{f},
$$

where $T_{\mathrm{ML} \sigma \mathrm{M}}^{f}$ corresponds to Eq. (A5) considering the whole fermionic content of the ML $\sigma \mathrm{M}$. The SM contribution to the $S$ parameter can be obtained by considering only the top and bottom contributions in Eq. (A7), and it turns out to be

$$
S_{\mathrm{SM}}^{f}=-\frac{1}{6 \pi} \log \left(\frac{m_{t}^{2}}{m_{b}^{2}}\right) \text {. }
$$

The deviation from the SM can be expressed in terms of the difference,

$$
\Delta S^{f}=S_{\mathrm{ML} \sigma \mathrm{M}}^{f}-S_{\mathrm{SM}}^{f},
$$

where $S_{\mathrm{ML} \sigma \mathrm{M}}^{f}$ is obtained from Eq. (A7) by considering the whole fermionic content of the ML $\sigma \mathrm{M}$.

The sum of scalar and fermionic contributions to both $T$ and $S$ must agree with experimental data,

$$
\begin{aligned}
& \Delta T \equiv T-T_{\mathrm{SM}}=0.06 \pm 0.06, \\
& \Delta S \equiv S-S_{\mathrm{SM}}=0.02 \pm 0.07,
\end{aligned}
$$

with a correlation of 0.92 .

\section{ML $\sigma$ M PARAMETER SPACE SCAN}

Here we discuss the details of our parameter scan of the $\mathrm{ML} \sigma \mathrm{M}$. For the mass and mixing of the new scalar, two benchmark points are chosen within the allowed region in Fig. 2,

$$
\begin{array}{ll}
m_{\sigma}=0.7 \mathrm{TeV}, & \sin ^{2} \gamma=0.1, \\
m_{\sigma}=1.0 \mathrm{TeV}, & \sin ^{2} \gamma=0.1 .
\end{array}
$$

In the fermionic sector, two different choices of parameters are considered:

(1) Both $y_{2}$ and $y_{2}^{\prime}$ are set to zero, since it is sufficient to have $\mathrm{y}_{1}$ and $\mathrm{y}_{1}^{\prime}$ different from zero to reproduce the measured values of the top and bottom quark masses at tree level. With $y_{2}=y_{2}^{\prime}=0, y_{1}$ and $y_{1}^{\prime}$ can be determined from other parameters and the quark masses in Eq. (33) which, up to second order, take the form

$$
\begin{aligned}
m_{t}= & \mathrm{y}_{1} \frac{v_{h}}{\sqrt{2}} \frac{\Lambda_{1} \Lambda_{3}}{M_{1} M_{5}}\left(1+\frac{\Lambda_{1}^{2}}{M_{5}^{2}}+\frac{\Lambda_{1}^{\prime 2}}{M_{5}^{\prime 2}}\right)^{-1 / 2} \\
& \times\left(1+\frac{\Lambda_{2}^{2}}{M_{5}^{2}}+\frac{\Lambda_{3}^{2}}{M_{1}^{2}}\right)^{-1 / 2},
\end{aligned}
$$

and similarly for the bottom sector, replacing the unprimed parameters with the primed ones and vice versa.

(2) A Yukawa coupling $y_{2}$ different from zero and relatively large $\left(\mathrm{y}_{2} \in[3.0,6.0]\right)$ is considered. The motivation for this choice is that, with this range of $\mathrm{y}_{2}$, the decay $T \rightarrow t \sigma$ has a quite sizable branching ratio as can be seen below.

For the remaining ML $\sigma \mathrm{M}$ parameters a numerical scan is performed. After numerically diagonalizing the mass matrices $\mathcal{M}_{\mathcal{T}}$ and $\mathcal{M}_{\mathcal{B}}$, the constraints discussed in Secs. III A-III C are enforced, such that all the model predictions for these observables agree with the experimental measurements at 95\% C.L. In addition to the above, the top quark mass resulting from the numerical diagonalization is required to be in the interval $[172,174] \mathrm{GeV}$, and the bottom quark mass in the interval $[4.4,4.8] \mathrm{GeV}$. At the same time, all Yukawa couplings are required to be far from the nonperturbativity limit $\left(\mathrm{y}_{1}, \mathrm{y}_{1}^{\prime}<6\right)$. The combination of all constraints can be satisfied for $\mathrm{M}_{i}, \mathrm{M}_{i}^{\prime}, \Lambda_{i}$ around a few $\mathrm{TeV}$ and $\Lambda_{i}^{\prime}$ around a few hundred GeV.

In the next section, the results of this scan are presented with a focus on the salient phenomenology of the model. In particular, we explore the relevant phenomenological 
features of the lightest exotic quarks, which generally dominate the phenomenology of $\mathrm{CH}$ scenarios as discussed previously in this work.

\section{LIGHTEST VLQ PHENOMENOLOGY}

As it is well known, VLQs can be produced at colliders like the LHC either in pairs or via single production. Examples of Feynman diagrams representing these two possibilities are shown in Fig. 3. In this work, the focus is on scenarios where the lightest VLQ has electric charge $2 / 3$ or $-1 / 3$, since it is in this case where the phenomenological features of the ML $\sigma \mathrm{M}$ may be qualitatively different than those of minimal $\mathrm{CH}$ models. Once produced, such a lightest VLQ state may decay into a thirdgeneration SM quark and a scalar or gauge boson [75].

The Lagrangian terms involved in the production and decay of the lightest exotic quarks of charge $2 / 3$ ( $T$ for the top partner) and $-1 / 3$ ( $B$ for the bottom partner) read

$$
\begin{aligned}
\mathscr{L}_{G}= & g_{s}\left(\bar{T} \gamma^{\mu} T+\bar{B} \gamma^{\mu} B\right) \frac{\lambda^{a}}{2} G_{\mu}^{a}, \\
\mathscr{L}_{W Q q}= & \frac{g}{\sqrt{2}}\left[\bar{T} \gamma^{\mu}\left(V_{T b}^{L} P_{L}+V_{T b}^{R} P_{R}\right) b\right. \\
& \left.+\bar{t} \gamma^{\mu}\left(V_{t B}^{L} P_{L}+V_{t B}^{R} P_{R}\right) B\right] W_{\mu}^{+}+\text {H.c., } \\
\mathscr{L}_{Z Q q}= & \frac{g}{2 c_{W}}\left[\bar{t} \gamma^{\mu}\left(X_{t T}^{L} P_{L}+X_{t T}^{R} P_{R}\right) T+\right. \\
& \left.-\bar{b} \gamma^{\mu}\left(X_{b B}^{L} P_{L}+X_{b B}^{R} P_{R}\right) B\right] Z_{\mu}+\text { H.c., } \\
\mathscr{L}_{h Q q}= & {\left[\bar{t}\left(y_{t T}^{h, L} P_{L}+y_{t T}^{h, R} P_{R}\right) T\right.} \\
& \left.+\bar{b}\left(y_{b B}^{h, L} P_{L}+y_{b B}^{h, R} P_{R}\right) B\right] h+\text { H.c., } \\
\mathscr{L}_{\sigma Q q}= & {\left[\bar{t}\left(y_{t T}^{\sigma, L} P_{L}+y_{t T}^{\sigma, R} P_{R}\right) T\right.} \\
& \left.+\bar{b}\left(y_{b B}^{\sigma, L} P_{L}+y_{b B}^{\sigma, R} P_{R}\right) B\right] \sigma+\text { H.c., }
\end{aligned}
$$

where $\lambda^{a}$ are the Gell-Mann matrices, $a$ is a color index, $G_{\mu}^{a}$ are the gluon fields and $g_{s}$ is the $S U(3)_{c}$ gauge coupling. In terms of the $V$ matrices introduced in Eq. (35),

$$
\begin{aligned}
V_{T b}^{L, R} & =\left(V_{L, R}\right)_{3,8}=\left(U_{L, R}^{\mathcal{T}} \mathcal{V}_{L, R}^{\mathcal{T} \mathcal{B}}\left(U_{L, R}^{\mathcal{B}}\right)^{\dagger}\right)_{2,1}, \\
V_{t B}^{L, R} & =\left(V_{L, R}\right)_{2,9}=\left(U_{L, R}^{\mathcal{T}} \mathcal{V}_{L, R}^{\mathcal{T} \mathcal{B}}\left(U_{L, R}^{\mathcal{B}}\right)^{\dagger}\right)_{1,2},
\end{aligned}
$$

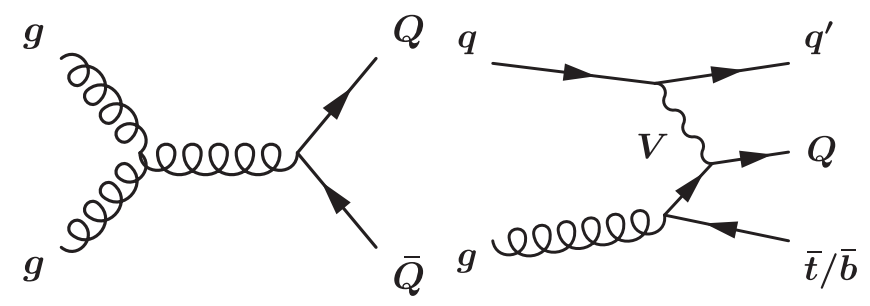

FIG. 3. Feynman diagrams for pair (left) and single (right) production of the VLQ state $Q$. For single VLQ production, $q$ and $q^{\prime}$ stand for generic SM quarks of the first two generations. where $U_{L, R}^{\mathcal{T}, \mathcal{B}}$ are the rotation matrices given in Eq. (30) and $\mathcal{V}_{L, R}^{\mathcal{T} \mathcal{B}}$ are the matrices defined in Eqs. (37) and (38). In terms of the $C$ matrices given in Eq. (39),

$$
\begin{aligned}
X_{t T}^{L, R} & =\left(C_{L, R}\right)_{2,3}=\left(U_{L, R}^{\mathcal{T}} \mathcal{C}_{L, R}^{\mathcal{T}}\left(U_{L, R}^{\mathcal{T}}\right)^{\dagger}\right)_{1,2}, \\
-X_{b B}^{L, R} & =\left(C_{L, R}\right)_{8,9}=\left(U_{L, R}^{\mathcal{B}} \mathcal{C}_{L, R}^{\mathcal{B}}\left(U_{L, R}^{\mathcal{B}}\right)^{\dagger}\right)_{1,2},
\end{aligned}
$$

where $\mathcal{C}_{L, R}^{\mathcal{T}, \mathcal{B}}$ are defined in Eq. (41). Finally, the Yukawa couplings of Eq. (64) are given by

$$
\begin{aligned}
& y_{t T}^{s, L}=\left(\mathrm{Y}_{s}\right)_{3,2}=\left(U_{L}^{\mathcal{T}} \mathscr{Y}_{s}^{\mathcal{T}}\left(U_{R}^{\mathcal{T}}\right)^{\dagger}\right)_{2,1}, \\
& y_{t T}^{s, R}=\left(\mathrm{Y}_{s}\right)_{2,3}=\left(U_{L}^{\mathcal{T}} \mathscr{Y}_{s}^{\mathcal{T}}\left(U_{R}^{\mathcal{T}}\right)^{\dagger}\right)_{1,2}, \\
& y_{b B}^{s, L}=\left(\mathrm{Y}_{s}\right)_{9,8}=\left(U_{L}^{\mathcal{B}} \mathscr{Y}_{s}^{\mathcal{B}}\left(U_{R}^{\mathcal{B}}\right)^{\dagger}\right)_{2,1}, \\
& y_{b B}^{s, R}=\left(\mathrm{Y}_{s}\right)_{8,9}=\left(U_{L}^{\mathcal{B}} \mathcal{Y}_{s}^{\mathcal{B}}\left(U_{R}^{\mathcal{B}}\right)^{\dagger}\right)_{1,2},
\end{aligned}
$$

with $s$ being either $h$ or $\sigma$, and $\mathrm{Y}_{s}$ is given in Eq. (43) and $\mathscr{Y}_{s}^{\mathcal{T}, \mathcal{B}}$ in Eq. (44).

In the following we consider separately the scenarios where the lightest VLQ has charge $-1 / 3(B)$ or charge $2 / 3(T)$.

\section{A. Bottom partner $(B)$ phenomenology}

For scenarios with the $B$ state as the lightest VLQ the most salient phenomenological feature is the large branching ratio $\operatorname{BR}(B \rightarrow \sigma b)$ that is possible in wide regions of the parameter space. This nonstandard VLQ decay may even be the dominant one. In Fig. 4, the results of our

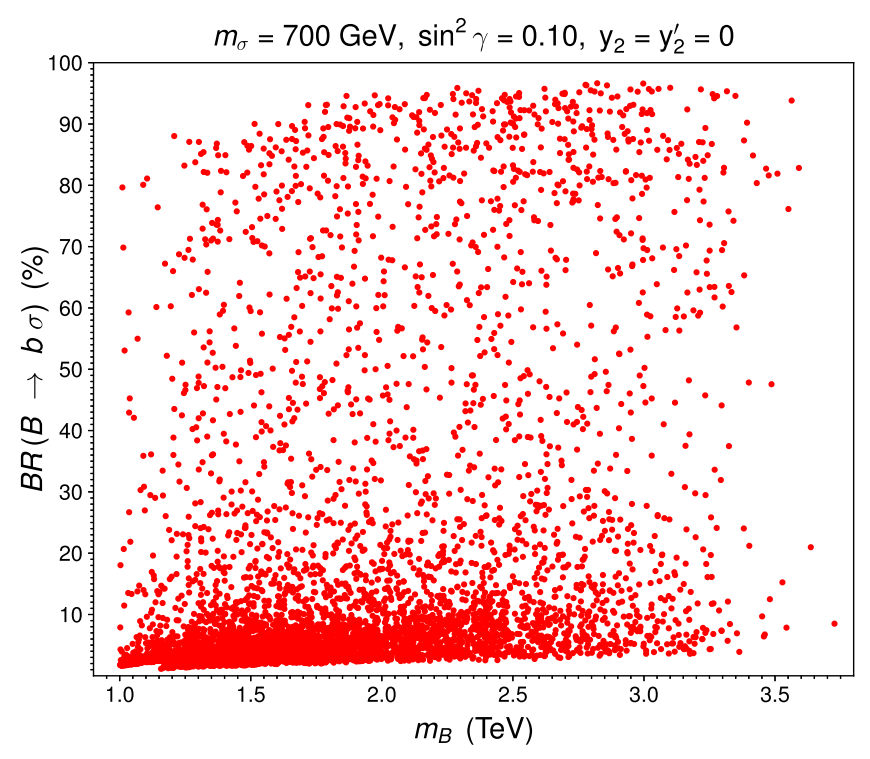

FIG. 4. Values of the branching ratio $\mathrm{BR}(B \rightarrow \sigma b)$ as a function of the bottom partner mass $m_{B}$ for the allowed points from our $\mathrm{ML} \sigma \mathrm{M}$ parameter scan (only points where $B$ is the lightest VLQ are included). In all these points the branching ratios of $\sigma$ are approximately $\operatorname{BR}\left(\sigma \rightarrow W^{+} W^{-}\right) \sim 40 \%, \operatorname{BR}(\sigma \rightarrow Z Z) \sim 30 \%$, $\operatorname{BR}(\sigma \rightarrow h h) \sim 20 \%$ and $\operatorname{BR}(\sigma \rightarrow t \bar{t}) \sim 10 \%$. 


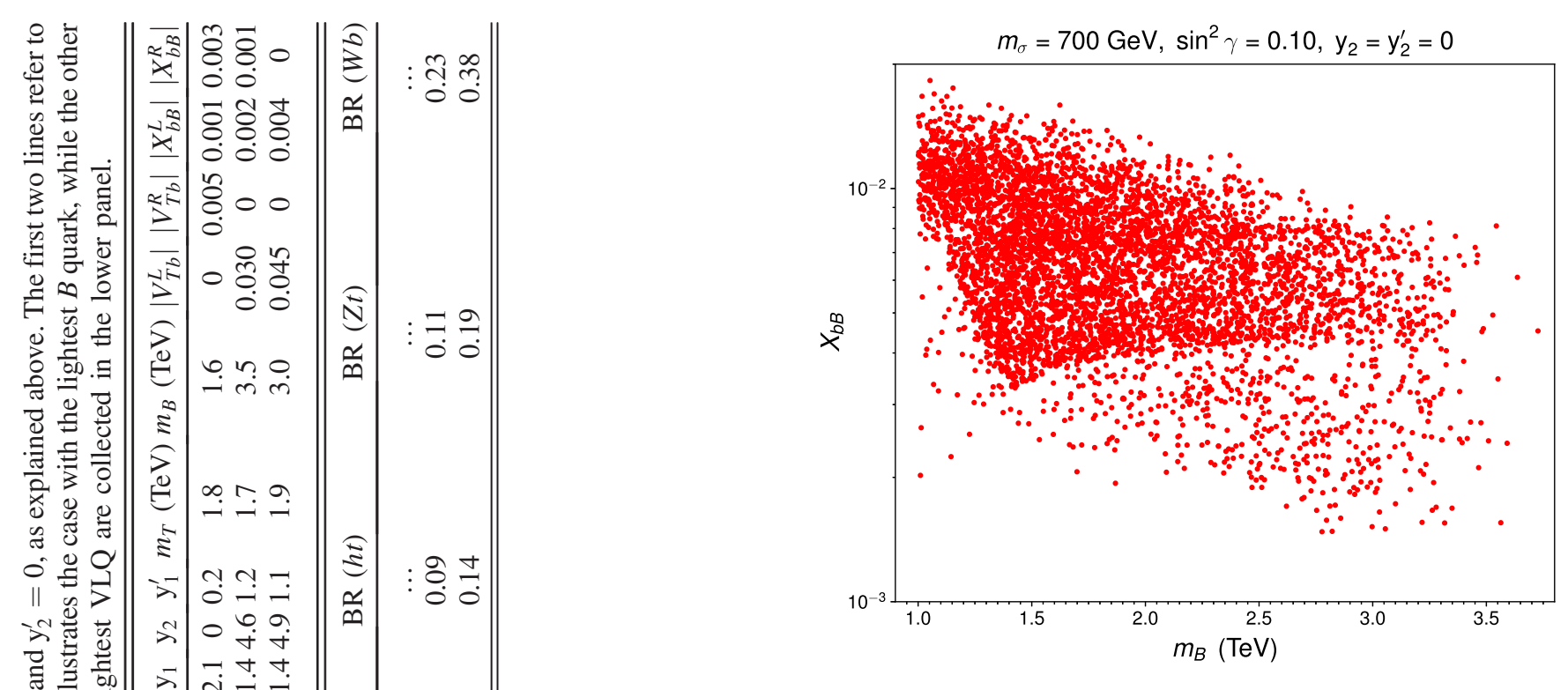

FIG. 5. Values of the $X_{b B}$ coupling in Eq. (68) as a function of the bottom partner mass $m_{B}$ for the allowed points from our $\mathrm{ML} \sigma \mathrm{M}$ parameter scan (only points where $B$ is the lightest VLQ are included).

$\mathrm{ML} \sigma \mathrm{M}$ scan for $m_{\sigma}=700 \mathrm{GeV}$ and $\mathrm{y}_{2}=\mathrm{y}_{2}^{\prime}=0$ are shown for $\operatorname{BR}(B \rightarrow \sigma b)$ as a function of the bottom partner mass $m_{B}$ (the varying density of points has no physical meaning but is rather an artifact of the parametrization used in the scan), showing that values $\operatorname{BR}(B \rightarrow \sigma b)>0.5$ may easily be obtained. The corresponding results for $m_{\sigma}=$ $1 \mathrm{TeV}$ are found to be quantitatively very similar. A specific example of model parameters that result in the lightest VLQ being a bottom partner state $B$ predominantly decaying into $\sigma b$ is given in the first line of Table III.

The dominant contribution to the single $B$ production cross section is given by the processes ${ }^{2} p p \rightarrow B \bar{b} q, p p \rightarrow$ $\bar{B} b q$ [through the Feynman diagram in Fig. 3 (right), with $V$ being the $Z$ boson], which are controlled by the strength of the $B b Z$ flavor-changing neutral coupling defined in terms of the couplings from Eq. (64) as

$$
X_{b B}=\sqrt{\left(X_{b B}^{L}\right)^{2}+\left(X_{b B}^{R}\right)^{2}} .
$$

Figure 5 shows the size of $X_{b B}$ as a function of $m_{B}$ for our ML $\sigma \mathrm{M}$ scan parameter points with $m_{\sigma}=700 \mathrm{GeV}$ and $\mathrm{y}_{2}=\mathrm{y}_{2}^{\prime}=0$. The values for $X_{b B}$ barely exceed 0.02 as seen in Fig. 5, resulting in single production cross sections for $B$ that are much smaller than the pair production cross section $\sigma(p p \rightarrow B \bar{B})$ at $\sqrt{s}=13 \mathrm{TeV}$, except for very large mass values $m_{B} \gtrsim 3.5 \mathrm{TeV}$. This can be seen explicitly in Fig. 6, which shows the $B$ pair production cross section

\footnotetext{
${ }^{2}$ Other single $B$ production channels like $p p \rightarrow B \bar{t} q$ and $p p \rightarrow \bar{B} t q$, proportional to $V_{t B}^{2}=\left(V_{t B}^{L}\right)^{2}+\left(V_{t B}^{R}\right)^{2}$, yield a subdominant contribution.
} 


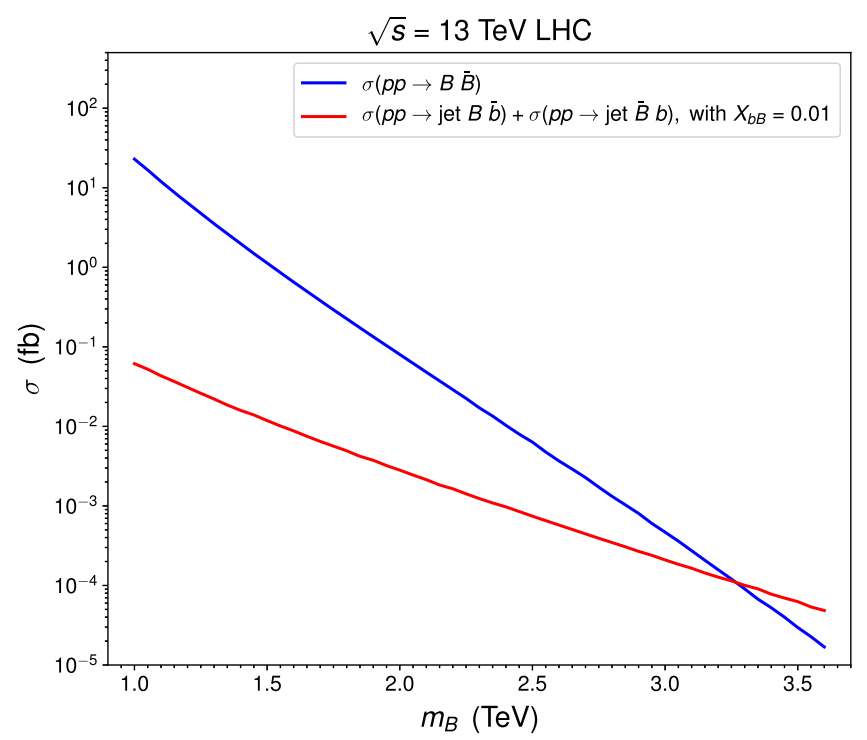

FIG. 6. Cross sections for $B$ pair production (blue) and single production with $X_{b B}=0.01$ (red) at the $13 \mathrm{TeV} \mathrm{LHC}$, as a function of the VLQ mass $m_{B}$.

(independent of $X_{b B}$ ) and the single $B$ production for $X_{b B}=0.01$, both obtained with Madgraph_aMC@NLO [76], using a FeynRules [77] implementation of the model in Eq. (64).

\section{B. Top partner $(T)$ phenomenology}

For scenarios with the $T$ state as the lightest VLQ, the branching ratio for the nonstandard decay $T \rightarrow \sigma t$ is generally small for $y_{2}=y_{2}^{\prime}=0$ but can be quite large for sizable $\mathrm{y}_{2}$, as shown in Fig. 7 respectively for $m_{\sigma}=$ $700 \mathrm{GeV}$ (upper panel) and $m_{\sigma}=1 \mathrm{TeV}$ (lower panel). The increase in $m_{\sigma}$ generically leads however to a decrease in the maximum possible size for $\operatorname{BR}(T \rightarrow \sigma t)$, as seen by comparing the upper and lower panels of Fig. 7 . The second and third lines of Table III respectively give an example of model parameters that result in the lightest VLQ being a $T$ quark with a large branching ratio to $\sigma t$ for $m_{\sigma}=700 \mathrm{GeV}$ and $m_{\sigma}=1 \mathrm{TeV}$.

The mixing of the $T$ state with the SM top and bottom quarks can be sizable in these scenarios, leading to large values of the single $T$ production cross section, but this is anticorrelated with the presence of a large $\operatorname{BR}(T \rightarrow \sigma t)$ : for large mixing the branching ratio to $\sigma t$ is smaller, and vice versa. Figure 8 shows the allowed values for the coupling $V_{T b}$, given in terms of the couplings from Eq. (64) as

$$
V_{T b}=\sqrt{\left(V_{T b}^{L}\right)^{2}+\left(V_{T b}^{R}\right)^{2}}
$$

as a function of the $T$ mass $m_{T}$. The different colors of the scan points correspond to different ranges of $\mathrm{BR}(T \rightarrow t \sigma)$. As Fig. 8 highlights, sizable mixings $V_{T b} \simeq 0.1$ are possible, but only for a small branching ratio for $T \rightarrow t \sigma$.
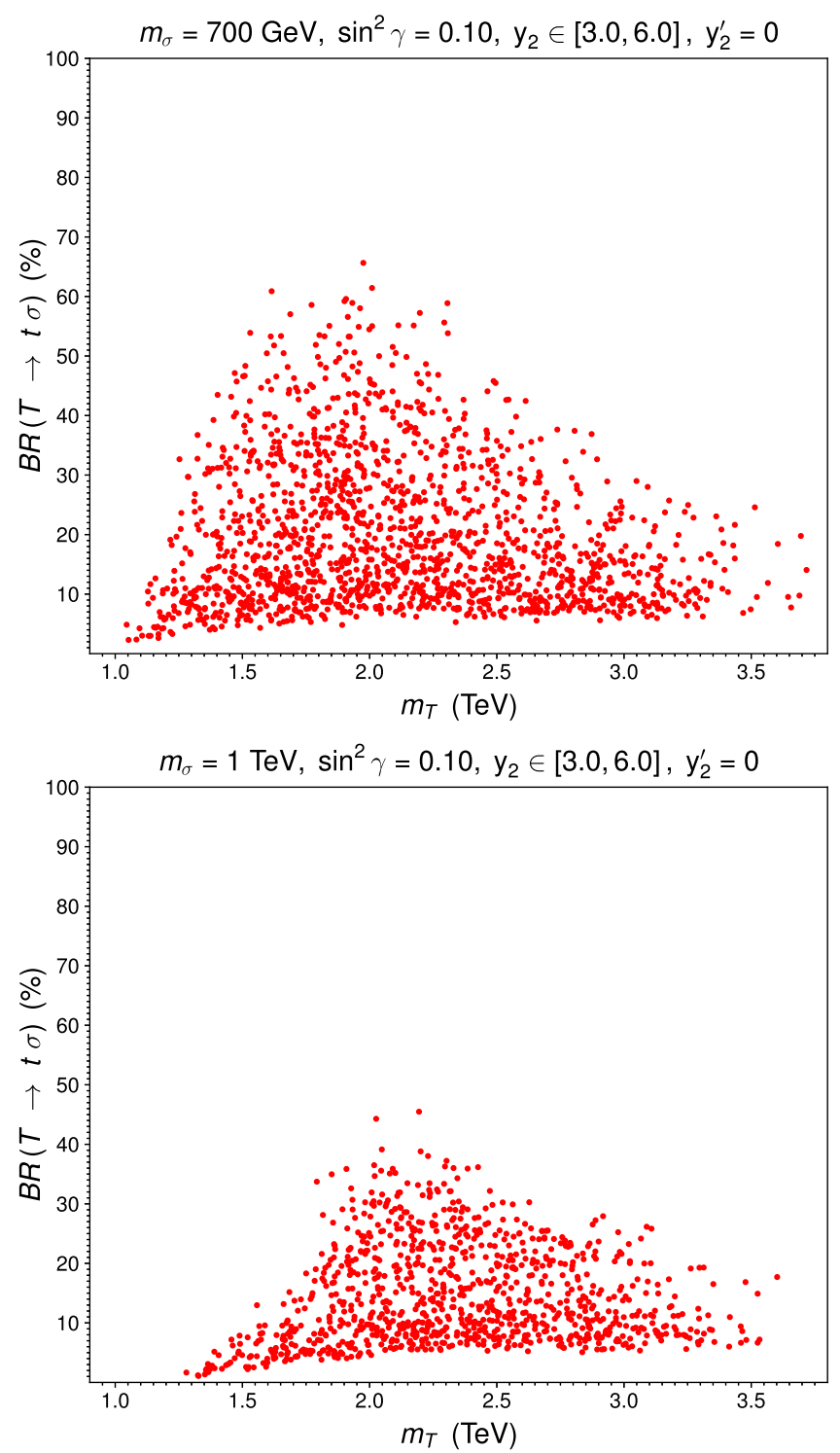

FIG. 7. Values of the branching ratio $\mathrm{BR}(T \rightarrow \sigma t)$ as a function of the top partner mass $m_{T}$ and $m_{\sigma}=700 \mathrm{GeV}$ (upper panel), $m_{\sigma}=1 \mathrm{TeV}$ (lower panel) for the allowed points from our ML $\sigma \mathrm{M}$ parameter scan (only points where $T$ is the lightest VLQ are included). At all these points the branching ratios of $\sigma$ are approximately $\mathrm{BR}\left(\sigma \rightarrow W^{+} W^{-}\right) \sim 40 \%, \mathrm{BR}(\sigma \rightarrow \mathrm{ZZ}) \sim 30 \%$, $\operatorname{BR}(\sigma \rightarrow h h) \sim 20 \%$ and $\operatorname{BR}(\sigma \rightarrow t \bar{t}) \sim 10 \%$.

Notice that there appears to be a minimum value of $V_{T b}$ as a function of $m_{T}: V_{T b}$ can indeed be below such apparent minima in Fig. 8, but in such a case a charge $-1 / 3 B$ state is lighter than $T$.

The coupling $V_{T b}$ controls the dominant contributions to single $T$ production via the processes $p p \rightarrow T \bar{b} q, p p \rightarrow$ $\bar{T} b q$ through the Feynman diagram in Fig. 3 (right), with $V$ being the $W$ boson. Figure 9 shows the $T$ pair production (independent of $V_{T b}$ ) and the single $T$ production cross sections for two benchmark values $V_{T b}=0.15,0.05$, computed with Madgraph_aMC@NLO [76]. It becomes clear 

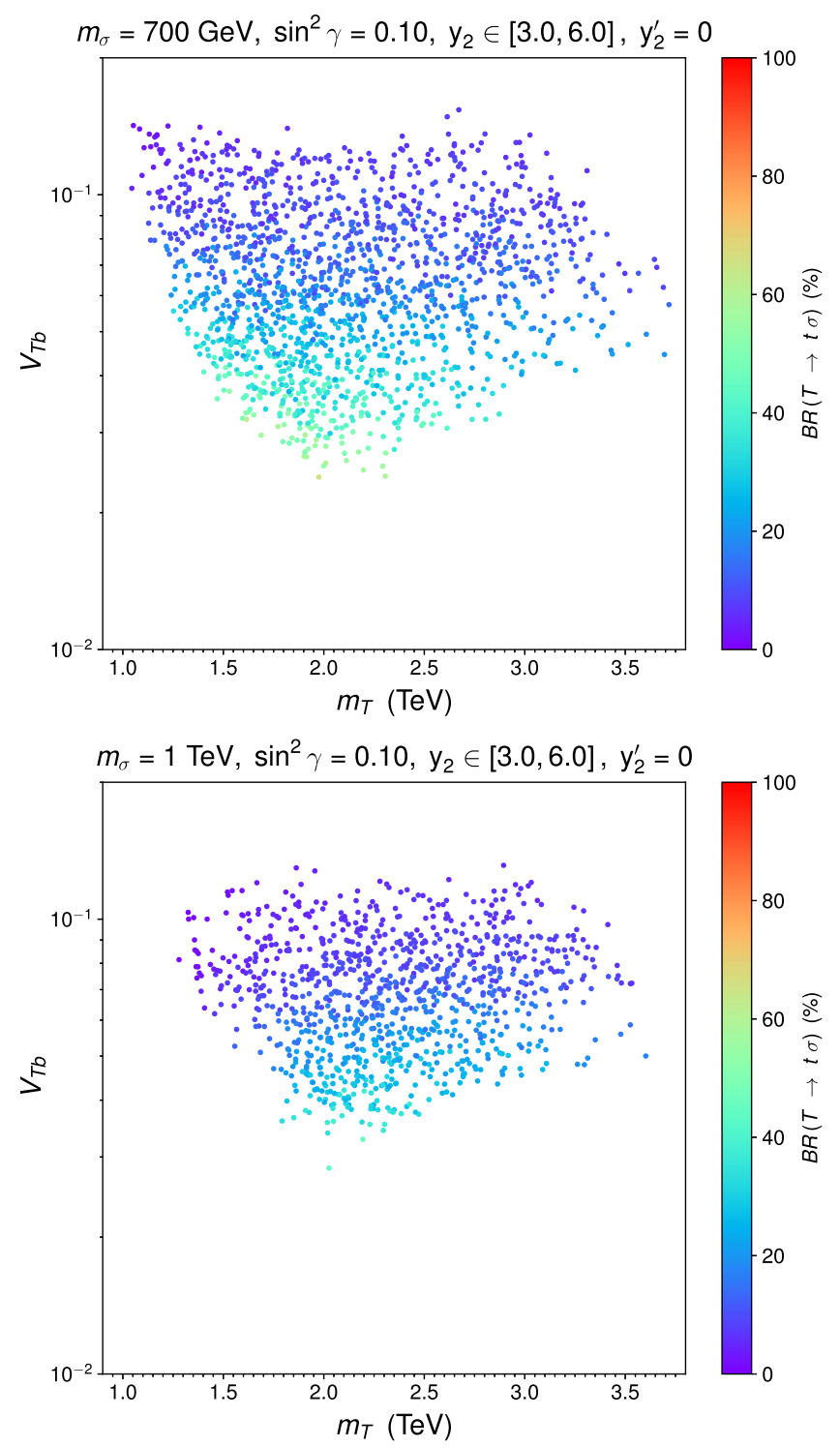

FIG. 8. Values of the $V_{T b}$ coupling in Eq. (69) as a function of the top partner mass $m_{T}$, for $\mathrm{y}_{2} \in[3.0,6.0]$ and respectively $m_{\sigma}=$ $700 \mathrm{GeV}$ (upper panel) and $m_{\sigma}=1 \mathrm{TeV}$ (upper panel), for the allowed points from our ML $\sigma \mathrm{M}$ parameter scan (only points where $B$ is the lightest VLQ are included). The colors of the scan points correspond to the range of values for $\operatorname{BR}(T \rightarrow \sigma t$ ) (see text for details).

that for $\mathcal{O}(0.1)$ mixings $V_{T b}^{L}$, the single $T$ production cross section becomes more important than the pair production one above $m_{T} \simeq 1 \mathrm{TeV}$.

Finally, let us comment that a general feature of models with more than just one VLQ state or multiplet is that the mixing of charge- $2 / 3$ exotic quarks $T$ with the thirdgeneration SM quarks can be larger than in minimal models [54]. As shown, this also happens in the ML $\sigma \mathrm{M}$ due to the interplay of different contributions to EWPOs from the several new fermions and the new scalar. From the experimental point of view, the possibility of large mixing

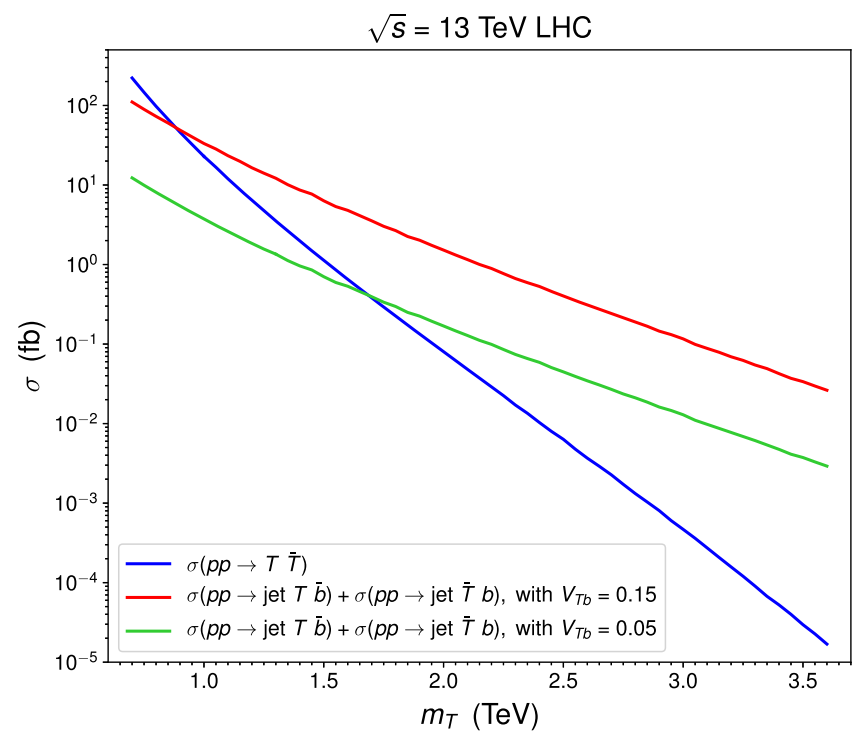

FIG. 9. $T$ pair production (blue) and single production for $V_{T b}=0.15$ (red) and $V_{T b}=0.05$ (green) cross sections at the $13 \mathrm{TeV}$ LHC, as a function of the VLQ mass $m_{T}$.

is very interesting, as it leads to much larger single $T$ production cross sections, which can in fact dominate the overall production of VLQ states above $m_{Q} \simeq 1 \mathrm{TeV}$.

\section{Current LHC sensitivity to $B \rightarrow \sigma b$ and $T \rightarrow \sigma t$}

As highlighted in the above sections, a salient characteristic of the ML $\sigma \mathrm{M}$ is the possibility of exotic decays of the new VLQ states, $B \rightarrow \sigma b$ and $T \rightarrow \sigma t$. The singlet state $\sigma$ dominantly decays into a pair of SM gauge bosons or a pair of $125 \mathrm{GeV}$ Higgses, the branching fraction into other SM states (e.g., $\sigma \rightarrow t \bar{t}$ ) being generally around 10\%. The exotic decays of the vectorlike $T, B$ quarks via Eqs. (2) and (3) then produce additional $W, Z$ or Higgs bosons compared to the standard VLQ decays in Eq. (1). In a resolved regime in which the $\sigma$ state is not very boosted (as a result of $m_{\sigma}$ and $m_{T / B}$ not being very far apart, which is something to be expected in the ML $\sigma \mathrm{M})$, its decay products are well separated and the signals resulting from single or pair production of VLQs followed by the exotic decays are similar to the ones already searched for at the LHC (i.e., the standard decays), but with higher SM gauge/Higgs boson multiplicities. It is then pertinent to ask ourselves to what extent the existing ATLAS and CMS searches are sensitive to the $B \rightarrow \sigma b$ and $T \rightarrow \sigma t$ decay signatures. The answer depends, case by case, on how inclusive the event selection for these experimental searches is. Several examples of LHC searches for VLQs that are sensitive to the new decay modes introduced in this work are as follows:

1. ATLAS search for same-sign dileptons or three leptons plus b-tagged jets [22]. This analysis primarily targets the process

$$
p p \rightarrow B \bar{B} \rightarrow W^{-} t W^{+} \bar{t} \rightarrow W^{-} W^{+} b W^{+} W^{-} \bar{b},
$$




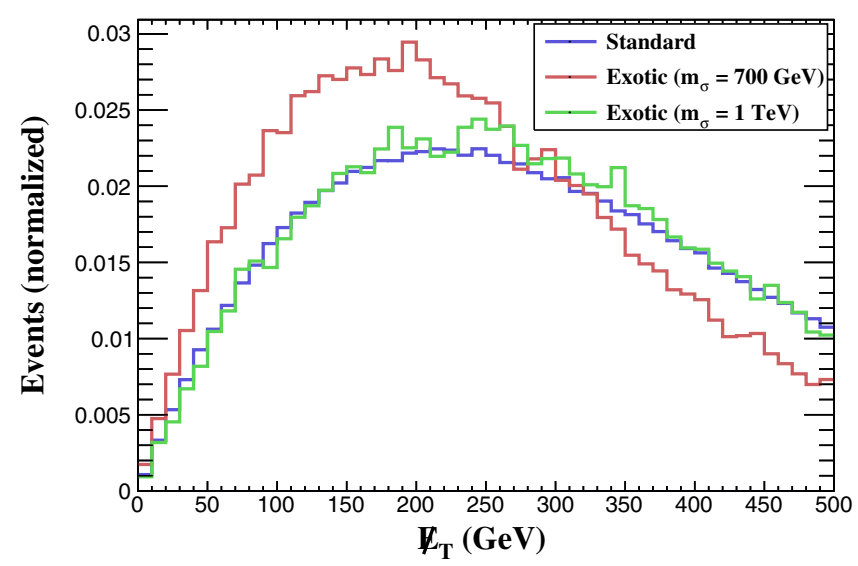

state contains two extra $W$ bosons compared to Eq. (70), and hence the combinatorial factor to get two same-sign leptons or three leptons is larger. The latter process yields the same final state as Eq. (70).

For illustration, Fig. 10 presents the normalized kinematical distributions of the missing transverse energy $\mathbb{E}_{T}$, transverse energy $H_{T}$ and leading lepton transverse momentum $\left(p_{T}\right)$, for the process in Eq. (70) with a standard VLQ decay mode $(B \rightarrow W t)$ and for the process $p p \rightarrow$ $B \bar{B} \rightarrow \sigma b \sigma \bar{b}$ with $\sigma \rightarrow W^{+} W^{-}$, respectively for $m_{\sigma}=$ $700 \mathrm{GeV}$ and $m_{\sigma}=1 \mathrm{TeV}$, as obtained at parton level using Madgraph_aMC@NLO [76] and MadAnalysis5 [78]. Notice that these distributions do not depend on the branching ratios of either the bottom partner $B$ or the heavy scalar $\sigma$. The similarity of the kinematical distributions makes it apparent that, despite being designed for a specific decay mode, the ATLAS search [22] is flexible enough to provide limits on other nonstandard decay modes of VLQ. In addition, we note that this search is also sensitive to single $T$ production followed by the exotic decay $T \rightarrow \sigma t$ $\left(\sigma \rightarrow W^{+} W^{-}\right)$:

$$
p p \rightarrow T b j, \quad T \rightarrow \sigma t \rightarrow W^{+} W^{-} W^{+} b,
$$

since two same-sign leptons or three leptons can be produced from the decay of a single $T$ state. Therefore, the same inclusive search can be used to probe both the mass and the coupling of new $T$ quarks with exotic decays.

2. ATLAS search for opposite-sign dileptons or three leptons plus b-tagged jets [21]. This analysis focuses on the decays of vectorlike quarks that involve a $Z$ boson,

$$
\begin{aligned}
& p p \rightarrow T+X \rightarrow Z t+X, \\
& p p \rightarrow B+X \rightarrow B b+X,
\end{aligned}
$$

with $X$ being additional particles, that is, another heavy VLQ in pair production or light SM quarks in single production. The event selection requires one same-flavor opposite-sign lepton pair with an invariant mass consistent with $M_{Z}$, as well as $b$-tagged jets and large $H_{T}$ (now defined with jets only). The analysis defines several signal regions according to the number of large-radius jets present with large mass. This search is sensitive to single and pair production of $T$ or $B$ quarks followed by the exotic decays in Eq. (2) and $\sigma \rightarrow Z Z$, with the advantage of the combinatorial factor stemming from the two $Z$ bosons that can decay leptonically.

3. ATLAS search for multijets with several b-tagged jets together with one charged lepton or large $\mathbb{E}_{T}$ [18]. This analysis focuses on pair production

$$
p p \rightarrow T \bar{T} \rightarrow h \stackrel{(-)}{t}+X,
$$

all transverse momenta of jets and leptons). This search is also sensitive to the processes $p p \rightarrow T \bar{T} \rightarrow \sigma t \sigma \bar{t}$ and $p p \rightarrow$ $B \bar{B} \rightarrow \sigma b \sigma \bar{b}$, with $\sigma \rightarrow W^{+} W^{-}$. In the former case, the final

that is, when either of the heavy quarks decays into $H \stackrel{(-)}{t}$ and the other one decays via any of the three standard VLQ 
decay modes in Eq. (1). Besides identifying top and Higgs candidates from the reconstructed objects, a large effective mass $m_{\text {eff }}=H_{T}+\mathbb{E}_{T}$ is required. This event selection is rather sensitive to pair production of $T$ states followed by the decay $T \rightarrow \sigma t$ with $\sigma \rightarrow h h$, where there are two Higgs bosons instead of only one as in the standard VLQ decay $T \rightarrow h t$.

With these examples one can easily understand that event selections that require high $H_{T}$ or $m_{\text {eff }}$ (characteristic of the production of heavy particles) and leptons or $b$ quarks are sensitive to-but not optimized for-the new VLQ decay modes discussed in this paper. The results of these searches can be reinterpreted by relaxing the conditions

$$
\begin{gathered}
\operatorname{Br}(T \rightarrow W b)+\operatorname{Br}(T \rightarrow Z t)+\operatorname{Br}(T \rightarrow h t)=1, \\
\operatorname{Br}(B \rightarrow W t)+\operatorname{Br}(B \rightarrow Z b)+\operatorname{Br}(B \rightarrow h b)=1,
\end{gathered}
$$

and introducing the additional decays in Eq. (2). On the other hand, there are searches that attempt to reconstruct the heavy quark masses by assuming a particular decay chain and kinematics $[15,20]$. Their sensitivity to the new modes in which either there are additional particles (e.g., in $T$ decays) or the kinematical configuration is different (in $B$ decays) is degraded.

\section{CONCLUSIONS}

Extensions of the SM that include new VLQs usually include extra new particles that may mediate new production or decay mechanisms for the VLQs. In this paper, we focused on the study of the phenomenology of the minimal linear $\sigma$ model, which is a renormalizable extension of the SM based on the global spontaneous symmetry breaking $S O(5) \rightarrow S O(4)$ that improves on several limitations of traditional composite Higgs models. The model includes several VLQs in the spectrum, as well as a new scalar $\sigma$ that can mediate novel decay channels for the VLQs.

A scan over the allowed parameter space shows that the new decay modes $T \rightarrow \sigma t, B \rightarrow \sigma b$ can have large branching ratios, and even dominate over the "standard" decays in Eq. (1). Moreover, the single production of $T$ quarks can be sizable and dominate over pair production, as a consequence of the interplay among the new VLQ states and the thirdgeneration SM quarks. This is a salient feature of realistic models (as opposed to simplified VLQ models) that is not generally possible with just one VLQ state or multiplet.
The pair and single productions of VLQs lead to final states with multiple bosons, e.g., $T \bar{T} \rightarrow \sigma t \sigma \bar{t} \rightarrow$ $W^{+} W^{-} W^{+} b W^{+} W^{-} W^{-} \bar{b}$. These new decay modes can in principle be captured by the current searches at the LHC. In this sense, the observability of such new decay modes requires that VLQ searches be rather generic, i.e., requiring the presence of multiple $b$ quarks, $W / Z / h$ bosons, high transverse energy, etc., and a few examples of ATLAS and CMS searches, which are sensitive to the new decay modes, have been discussed in this work, and can be straightforwardly interpreted to set limits on those decays.

To conclude, the possibility of new VLQ decays is open and demands more generic experimental analyses, with a wide sensitivity beyond the "standard" decays, and providing limits on the new modes. Dedicated searches for these new modes would benefit from the conspicuous multiboson signatures produced in these decays, and would also be welcome.

\section{ACKNOWLEDGMENTS}

J. A. G. and L. M. thanks Stefano Rigolin for useful discussions. The authors acknowledge partial financial support by the Spanish MINECO through the Centro de excelencia Severo Ochoa Program under Grant No. SEV2016-0597. J. A. G., L. M. and J. A. A. S. acknowledge partial financial support by the Spanish "Agencia Estatal de Investigación" (AEI) and the EU "Fondo Europeo de Desarrollo Regional" (FEDER) through the Projects No. FPA2016-78645-P and No. FPA2016-78220-C3-1-P. L. M. acknowledges partial financial support by the Spanish MINECO through the "Ramón y Cajal" programme (RYC-2015-17173). J. M. N. acknowledges support from the Ramón y Cajal Fellowship Contract No. RYC-2017-22986 and from the Spanish Proyectos de $\mathrm{I}+\mathrm{D}$ de Generación de Conocimiento via Grant No. PGC2018-096646-A-I00. L. M. and J. M. N. also acknowledge support from the European Union's Horizon 2020 research and innovation programme under the Marie Sklodowska-Curie Grant agreements No. 690575 (RISE InvisiblesPlus) and No. 674896 (ITN ELUSIVES).

\section{APPENDIX A: EXPRESSIONS FOR THE CORRECTIONS TO $S$ AND $T$ PARAMETERS}

The explicit expression for $\Delta T^{(h \text { and } \sigma)}$ is given by ${ }^{3}$

$$
\begin{aligned}
\Delta T^{(h \text { and } \sigma)}= & \frac{3 s_{\gamma}^{2}}{16 s_{W}^{2} \pi}\left[m_{h}^{2} \frac{\log \left(m_{h}^{2} / m_{W}^{2}\right)}{m_{W}^{2}-m_{h}^{2}}-m_{\sigma}^{2} \frac{\log \left(m_{\sigma}^{2} / m_{W}^{2}\right)}{m_{W}^{2}-m_{\sigma}^{2}}\right. \\
& \left.+\frac{m_{Z}^{2}}{m_{W}^{2}}\left(-m_{h}^{2} \frac{\log \left(m_{h}^{2} / m_{Z}^{2}\right)}{m_{Z}^{2}-m_{h}^{2}}+m_{\sigma}^{2} \frac{\log \left(m_{\sigma}^{2} / m_{Z}^{2}\right)}{m_{Z}^{2}-m_{\sigma}^{2}}\right)\right] .
\end{aligned}
$$

\footnotetext{
${ }^{3}$ This expression differs from the one in Eq. (51) of Ref. [31]: an $\alpha$ is missing multiplying $\Delta T^{(h \text { and } \sigma)}$ on the left-hand side of that expression, while a minus sign should be present on the right-hand side.
} 
On the other side, for the computation of $\Delta S^{(h \text { and } \sigma)}$, the explicit expression of $S_{\mathrm{SM}}^{h}$ can be found in Refs. $[79,80]^{4}$ :

$$
S_{\mathrm{SM}}^{h}(x)=\frac{1}{\pi}\left[\frac{x}{12(x-1)} \log (x)+\left(-\frac{x}{6}+\frac{x^{2}}{12}\right) F(x)-\left(1-\frac{x}{3}+\frac{x^{2}}{12}\right) F^{\prime}(x)\right],
$$

where $x \equiv m^{2} / m_{Z}^{2}$, and with the $F(x)$ and $F^{\prime}(x)$ functions given by

$$
\begin{aligned}
& F(x)=1+\left(\frac{x}{x-1}-\frac{x}{2}\right) \log (x)-x \sqrt{\frac{4}{x}-1} \arctan \left(\sqrt{\frac{4}{x}-1}\right) \\
& F^{\prime}(x)=-1+\frac{x-1}{2} \log (x)+(3-x) \sqrt{\frac{x}{4-x}} \arctan \left(\sqrt{\frac{4}{x}-1}\right)
\end{aligned}
$$

for $x<4$, whereas for $x>4^{5}$

$$
\begin{aligned}
& F(x)=1+\left(\frac{x}{x-1}-\frac{x}{2}\right) \log (x)+x \sqrt{1-\frac{4}{x}} \log \left(\sqrt{\frac{x}{4}-1}+\sqrt{\frac{x}{4}}\right), \\
& F^{\prime}(x)=-1+\frac{x-1}{2} \log (x)+(3-x) \sqrt{\frac{x}{x-4}} \log \left(\sqrt{\frac{x}{4}-1}+\sqrt{\frac{x}{4}}\right) .
\end{aligned}
$$

The generalized contribution to the $T$ parameter from VLQs with arbitrary couplings was presented in Ref. [73]:

$$
\begin{aligned}
T^{f}= & \frac{N_{c}}{16 \pi s_{W}^{2} c_{W}^{2}}\left(\sum_{i, j}\left[\left(\left|V_{L}^{i j}\right|^{2}+\left|V_{R}^{i j}\right|^{2}\right) \theta_{+}\left(x_{i}, x_{j}\right)+2 \operatorname{Re}\left(V_{L}^{i j} V_{R}^{i j *}\right) \theta_{-}\left(x_{i}, x_{j}\right)\right]+\right. \\
& \left.-\frac{1}{2} \sum_{i, j}\left[\left(\left|C_{L}^{i j}\right|^{2}+\left|C_{R}^{i j}\right|^{2}\right) \theta_{+}\left(x_{i}, x_{j}\right)+2 \operatorname{Re}\left(C_{L}^{i j} C_{R}^{i j *}\right) \theta_{-}\left(x_{i}, x_{j}\right)\right]\right),
\end{aligned}
$$

where $N_{c}$ is the number of colors, $x_{i} \equiv m_{i}^{2} / m_{Z}^{2}$ with $m_{i}$ being the VLQ masses, and $V_{L, R}$ and $C_{L, R}$ are the matrices given in Eqs. (35) and (39). The $\theta_{ \pm}$functions are defined as

$$
\begin{aligned}
& \theta_{+}\left(x_{1}, x_{2}\right) \equiv x_{1}+x_{2}-\frac{2 x_{1} x_{2}}{x_{1}-x_{2}} \log \left(\frac{x_{1}}{x_{2}}\right)-2\left(x_{1} \log \left(x_{1}\right)+x_{2} \log \left(x_{2}\right)\right)+\frac{x_{1}+x_{2}}{2} \Delta, \\
& \theta_{-}\left(x_{1}, x_{2}\right) \equiv 2 \sqrt{x_{1} x_{2}}\left(\frac{x_{1}+x_{2}}{x_{1}-x_{2}} \log \left(\frac{x_{1}}{x_{2}}\right)-2+\log \left(x_{1} x_{2}\right)-\frac{\Delta}{2}\right),
\end{aligned}
$$

where $\Delta$ is a divergent quantity arising in the dimensional regularization. This contribution disappears in the sum of all the contributions.

Similarly for the $S$ parameter, the generic expression can be found in Ref. [74] and reads

$$
S^{f}=\frac{N_{c}}{2 \pi}\left(\sum_{i, j}\left[\left(X_{L}^{i j *} Y_{L}^{i j}+X_{R}^{i j *} Y_{R}^{i j}\right) \chi_{+}\left(\hat{\mathcal{M}}_{i i}, \hat{\mathcal{M}}_{j j}\right)+\left(X_{L}^{i j *} Y_{R}^{i j}+X_{R}^{i j *} Y_{L}^{i j}\right) \chi_{-}\left(\hat{\mathcal{M}}_{i i}, \hat{\mathcal{M}}_{j j}\right)\right]\right),
$$

where here $X_{L, R}$ are the isospin-dependent coupling matrices in the mass basis [that is, the $U_{L, R} \mathcal{C}_{L, R} U_{L, R}^{\dagger}$ matrices present in Eq. (39)], the $Y_{L, R}$ are the hypercharge matrices of the fermions and the $\chi_{ \pm}$functions are defined as

$$
\begin{aligned}
& \chi_{+}\left(m_{1}, m_{2}\right)=\frac{5\left(m_{1}^{4}+m_{2}^{4}\right)-22 m_{1}^{2} m_{2}^{2}}{9\left(m_{1}^{2}-m_{2}^{2}\right)^{2}}+\frac{3 m_{1}^{2} m_{2}^{2}\left(m_{1}^{2}+m_{2}^{2}\right)-\left(m_{1}^{6}+m_{2}^{6}\right)}{3\left(m_{1}^{2}-m_{2}^{2}\right)^{3}} \log \left(\frac{m_{1}^{2}}{m_{2}^{2}}\right)-\frac{2}{3} \log \left(\frac{m_{1} m_{2}}{\mu^{2}}\right), \\
& \chi_{-}\left(m_{1}, m_{2}\right)=\frac{m_{1} m_{2}}{\left(m_{1}^{2}-m_{2}^{2}\right)^{3}}\left[m_{1}^{4}-m_{2}^{4}-2 m_{1}^{2} m_{2}^{2} \log \left(\frac{m_{1}^{2}}{m_{2}^{2}}\right)\right],
\end{aligned}
$$

\footnotetext{
${ }^{4} \mathrm{~A}$ typo is present in Eq. (F.7) of Ref. [79] that has been corrected in Ref. [81].

${ }^{5}$ Equation (55) of Ref. [31] disagrees by a minus sign with respect to Refs. [80,81]. The correct version is in Eq. (A4) which matches Refs. [80,81].
} 
where $\mu$ is an arbitrary scale that disappears as long as $\operatorname{Tr}\left[X_{L}^{\dagger} Y_{L}+X_{R}^{\dagger} Y_{R}\right]=0$.

In the ML $\sigma \mathrm{M}$, the hypercharge matrices are defined by

$$
Y_{L, R}=U_{L, R} \mathcal{Y}_{L, R} U_{L, R}^{\dagger}
$$

where the $\mathcal{Y}_{L, R}$ are given by

$$
\mathcal{Y}_{L, R}=\operatorname{diag}\left(\frac{7}{6}, \mathcal{Y}_{L, R}^{\mathcal{T}}, \mathcal{Y}_{L, R}^{\mathcal{B}},-\frac{5}{6}\right)
$$

with

$$
\begin{aligned}
& \mathcal{Y}_{L}^{\mathcal{T}}=\operatorname{diag}\left(\frac{1}{6}, \frac{1}{6}, \frac{7}{6}, \frac{2}{3}, \frac{2}{3}, \frac{1}{6}\right), \quad \mathcal{Y}_{R}^{\mathcal{T}}=\operatorname{diag}\left(\frac{2}{3}, \frac{1}{6}, \frac{7}{6}, \frac{2}{3}, \frac{2}{3}, \frac{1}{6}\right), \\
& \mathcal{Y}_{L}^{\mathcal{B}}=\operatorname{diag}\left(\frac{1}{6}, \frac{1}{6},-\frac{5}{6},-\frac{1}{3},-\frac{1}{3}, \frac{1}{6}\right), \quad \mathcal{Y}_{R}^{\mathcal{B}}=\operatorname{diag}\left(-\frac{1}{3}, \frac{1}{6},-\frac{5}{6},-\frac{1}{3},-\frac{1}{3}, \frac{1}{6}\right) .
\end{aligned}
$$

\section{APPENDIX B: EXPRESSIONS FOR THE PARTIAL WIDTHS}

The explicit computation of the decay rates leads to the following results. For the process involving a scalar,

$$
\Gamma_{\psi_{j} \rightarrow \psi_{i} s}=\frac{1}{32 \pi} \frac{\lambda^{1 / 2}\left(m_{j}^{2}, m_{i}^{2}, m_{s}^{2}\right)}{m_{j}}\left[\left(\left(g_{L}^{\psi s}\right)_{i j}^{2}+\left(g_{R}^{\psi s}\right)_{i j}^{2}\right)\left(1+\frac{m_{i}^{2}-m_{s}^{2}}{m_{j}^{2}}\right)+4\left(g_{L}^{\psi s}\right)_{i j}\left(g_{R}^{\psi s}\right)_{i j} \frac{m_{i}}{m_{j}}\right]
$$

with $s$ being either $h$ or $\sigma, \psi_{i, j}$ are the components of $\hat{\mathcal{T}}$ or $\hat{\mathcal{B}}$ of Eq. (32), the couplings $g_{L, R}^{\psi s}$ are defined in Table IV and $\lambda$ is given by

$$
\lambda=\left[m_{j}^{2}-\left(m_{i}^{2}+m_{s}^{2}\right)^{2}\right]\left[m_{j}^{2}-\left(m_{i}^{2}-m_{s}^{2}\right)^{2}\right] .
$$

\begin{tabular}{|c|c|}
\hline & $\left(g_{L, R}\right)_{i j}$ \\
\hline$\psi_{j} \rightarrow \psi_{i} s$ & $\begin{array}{l}\left(g_{L}^{\psi s}\right)_{i j}=\left(U_{R}^{\psi} \mathscr{Y}_{s}^{\psi^{\dagger} \dagger} U_{L}^{\psi^{\dagger}}\right)_{i j} \\
\left(g_{R}^{\psi s}\right)_{i j}=\left(U_{L}^{\psi} \mathcal{Y}_{s}^{\psi} U_{R}^{\psi^{\dagger} \dagger}\right)_{i j}\end{array}$ \\
\hline$\hat{\mathcal{T}}_{j} \rightarrow K^{u} W^{-}$ & $\left(g_{L, R}\right)_{i j}=\left(U_{L, R}^{\mathcal{T} \dagger}\right)_{3 j}$ \\
\hline$\hat{\mathcal{B}}_{j} \rightarrow K^{\prime d} W^{+}$ & $\left(g_{L, R}\right)_{i j}=\left(U_{L, R}^{\mathcal{B} \dagger}\right)_{3 j}$ \\
\hline$\hat{\mathcal{T}}_{j} \rightarrow \hat{\mathcal{B}}_{i} W^{+}$ & $\left(g_{L, R}\right)_{i j}=\left(U_{L, R}^{\mathcal{B}} \mathcal{V}_{L, R}^{\mathcal{T} \mathcal{B}} U_{L, R}^{\mathcal{T} \dagger}\right)_{i j}$ \\
\hline$\psi_{j} \rightarrow \psi_{i} Z$ & $\left(g_{L, R}\right)_{i j}=\left(C_{L, R}\right)_{i j}$ \\
\hline
\end{tabular}

Instead, for a process with a gauge boson,

$$
\begin{aligned}
\Gamma_{\psi_{j} \rightarrow \psi_{i}^{\prime} V}= & \frac{g^{2}}{64 \pi} k_{V} \frac{m_{j}}{m_{V}^{2}} \lambda^{1 / 2}\left(m_{j}^{2}, m_{i}^{2}, m_{V}^{2}\right) \\
& \times\left[\left(\left(g_{L}\right)_{i j}^{2}+\left(g_{R}\right)_{i j}^{2}\right)\left(1+\frac{m_{V}^{2}-2 m_{i}^{2}}{m_{j}^{2}}+\frac{m_{i}^{4}+m_{i}^{2} m_{V}^{2}-2 m_{V}^{4}}{m_{j}^{4}}\right)-12\left(g_{L}\right)_{i j}\left(g_{R}\right)_{i j} \frac{m_{i} m_{V}^{2}}{m_{j}^{3}}\right],
\end{aligned}
$$

with $k_{V}=1$ for $V=W^{ \pm}$and $k_{V}=1 /\left(2 c_{W}^{2}\right)$ for $V=Z$ and the couplings are given again in Table IV.

TABLE IV. Couplings entering the decay rates in Eqs. (B2) and (B3). 
[1] D. B. Kaplan and H. Georgi, $S U(2) \times U(1)$ breaking by vacuum misalignment, Phys. Lett. 136B, 183 (1984).

[2] D. B. Kaplan, H. Georgi, and S. Dimopoulos, Composite Higgs scalars, Phys. Lett. 136B, 187 (1984).

[3] T. Banks, Constraints on $S U(2) \times U(1)$ breaking by vacuum misalignment, Nucl. Phys. B243, 125 (1984).

[4] K. Agashe, R. Contino, and A. Pomarol, The minimal composite Higgs model, Nucl. Phys. B719, 165 (2005).

[5] R. Barbieri, B. Bellazzini, V. S. Rychkov, and A. Varagnolo, The Higgs boson from an extended symmetry, Phys. Rev. D 76, 115008 (2007).

[6] B. Gripaios, A. Pomarol, F. Riva, and J. Serra, Beyond the minimal composite Higgs model, J. High Energy Phys. 04 (2009) 070.

[7] J. Mrazek, A. Pomarol, R. Rattazzi, M. Redi, J. Serra, and A. Wulzer, The other natural two Higgs doublet model, Nucl. Phys. B853 (2011) 1.

[8] M. J. Dugan, H. Georgi, and D. B. Kaplan, Anatomy of a composite Higgs model, Nucl. Phys. B254, 299 (1985).

[9] D. B. Kaplan, Flavor at Ssc Energies: A new mechanism for dynamically generated fermion masses, Nucl. Phys. B365, 259 (1991).

[10] R. Contino and A. Pomarol, Holography for fermions, J. High Energy Phys. 11 (2004) 058.

[11] P. Minkowski, $\mu \rightarrow e \gamma$ at a rate of one out of $10^{9}$ muon decays?, Phys. Lett. 67B, 421 (1977).

[12] S. Weinberg, Baryon and Lepton Nonconserving Processes, Phys. Rev. Lett. 43, 1566 (1979).

[13] T. Yanagida, Horizontal symmetry and masses of neutrinos, Prog. Theor. Phys. 64, 1103 (1980).

[14] M. Aaboud et al. (ATLAS Collaboration), Search for pair production of vector-like top quarks in events with one lepton, jets, and missing transverse momentum in $\sqrt{s}=$ $13 \mathrm{TeV} p p$ collisions with the ATLAS detector, J. High Energy Phys. 08 (2017) 052.

[15] M. Aaboud et al. (ATLAS Collaboration), Search for pair production of heavy vector-like quarks decaying to high- $\mathrm{p}_{T}$ $\mathrm{W}$ bosons and $\mathrm{b}$ quarks in the lepton-plus-jets final state in pp collisions at $\sqrt{s}=13 \mathrm{TeV}$ with the ATLAS detector, J. High Energy Phys. 10 (2017) 141.

[16] A. M. Sirunyan et al. (CMS Collaboration), Search for pair production of vector-like quarks in the $\mathrm{bW} \overline{\mathrm{b}} \mathrm{W}$ channel from proton-proton collisions at $\sqrt{s}=13 \mathrm{TeV}$, Phys. Lett. B 779, 82 (2018).

[17] A. M. Sirunyan et al. (CMS Collaboration), Search for single production of vector-like quarks decaying to a B quark and a Higgs boson, J. High Energy Phys. 06 (2018) 031.

[18] M. Aaboud et al. (ATLAS Collaboration), Search for pair production of up-type vector-like quarks and for four-topquark events in final states with multiple $b$-jets with the ATLAS detector, J. High Energy Phys. 07 (2018) 089.

[19] A. M. Sirunyan et al. (CMS Collaboration), Search for vector-like $\mathrm{T}$ and $\mathrm{B}$ quark pairs in final states with leptons at $\sqrt{s}=13 \mathrm{TeV}$, J. High Energy Phys. 08 (2018) 177.

[20] M. Aaboud et al. (ATLAS Collaboration), Search for pair production of heavy vector-like quarks decaying into high- $p_{T} W$ bosons and top quarks in the lepton-plus-jets final state in $p p$ collisions at $\sqrt{s}=13 \mathrm{TeV}$ with the ATLAS detector, J. High Energy Phys. 08 (2018) 048.
[21] M. Aaboud et al. (ATLAS Collaboration), Search for pairand single-production of vector-like quarks in final states with at least one $Z$ boson decaying into a pair of electrons or muons in $p p$ collision data collected with the ATLAS detector at $\sqrt{s}=13 \mathrm{TeV}$, Phys. Rev. D 98, 112010 (2018).

[22] M. Aaboud et al. (ATLAS Collaboration), Search for new phenomena in events with same-charge leptons and $b$-jets in $p p$ collisions at $\sqrt{s}=13 \mathrm{TeV}$ with the ATLAS detector, J. High Energy Phys. 12 (2018) 039.

[23] M. Aaboud et al. (ATLAS Collaboration), Search for pair production of heavy vector-like quarks decaying into hadronic final states in $p p$ collisions at $\sqrt{s}=13 \mathrm{TeV}$ with the ATLAS detector, Phys. Rev. D 98, 092005 (2018).

[24] A. M. Sirunyan et al. (CMS Collaboration), Search for single production of vector-like quarks decaying to a top quark and a $\mathrm{W}$ boson in proton-proton collisions at $\sqrt{s}=13 \mathrm{TeV}$, Eur. Phys. J. C 79, 90 (2019).

[25] A. M. Sirunyan et al. (CMS Collaboration), Search for top quark partners with charge $5 / 3$ in the same-sign dilepton and single-lepton final states in proton-proton collisions at $\sqrt{s}=13 \mathrm{TeV}$, J. High Energy Phys. 03 (2019) 082.

[26] M. Aaboud et al. (ATLAS Collaboration), Search for single production of vector-like quarks decaying into $W b$ in $p p$ collisions at $\sqrt{s}=13 \mathrm{TeV}$ with the ATLAS detector, J. High Energy Phys. 05 (2019) 164.

[27] M. Aaboud et al. (ATLAS Collaboration), Search for large missing transverse momentum in association with one topquark in proton-proton collisions at $\sqrt{s}=13 \mathrm{TeV}$ with the ATLAS detector, J. High Energy Phys. 05 (2019) 041.

[28] A. M. Sirunyan et al. (CMS Collaboration), Search for vector-like quarks in events with two oppositely charged leptons and jets in proton-proton collisions at $\sqrt{s}=13 \mathrm{TeV}$, Eur. Phys. J. C 79, 364 (2019).

[29] A. M. Sirunyan et al. (CMS Collaboration), Search for pair production of vectorlike quarks in the fully hadronic final state, Phys. Rev. D 100, 072001 (2019).

[30] A. M. Sirunyan et al. (CMS Collaboration), Search for electroweak production of a vector-like $\mathrm{T}$ quark using fully hadronic final states, J. High Energy Phys. 01 (2020) 036.

[31] F. Feruglio, B. Gavela, K. Kanshin, P. A. N. Machado, S. Rigolin, and S. Saa, The minimal linear sigma model for the goldstone Higgs, J. High Energy Phys. 06 (2016) 038.

[32] R. Alonso, I. Brivio, B. Gavela, L. Merlo, and S. Rigolin, Sigma decomposition, J. High Energy Phys. 12 (2014) 034.

[33] G. Panico and A. Wulzer, The composite Nambu-Goldstone Higgs, Lect. Notes Phys. 913, 1 (2016).

[34] I. M. Hierro, L. Merlo, and S. Rigolin, Sigma decomposition: The Cp-odd Lagrangian, J. High Energy Phys. 04 (2016) 016.

[35] M. B. Gavela, K. Kanshin, P. A. N. Machado, and S. Saa, The linear-non-linear frontier for the Goldstone Higgs, Eur. Phys. J. C 76, 690 (2016).

[36] F. Feruglio, The chiral approach to the electroweak interactions, Int. J. Mod. Phys. A 08, 4937 (1993).

[37] B. Grinstein and M. Trott, A Higgs-Higgs bound state due to new physics at a TeV, Phys. Rev. D 76, 073002 (2007).

[38] R. Contino, C. Grojean, M. Moretti, F. Piccinini, and R. Rattazzi, Strong double Higgs production at the Lhc, J. High Energy Phys. 05 (2010) 089. 
[39] R. Alonso, M. B. Gavela, L. Merlo, S. Rigolin, and J. Yepes, The effective chiral lagrangian for a light dynamical "Higgs particle", Phys. Lett. B 722, 330 (2013); Erratum, Phys. Lett. B 726, 926 (2013).

[40] R. Alonso, M. B. Gavela, L. Merlo, S. Rigolin, and J. Yepes, Flavor with a light dynamical "Higgs particle", Phys. Rev. D 87, 055019 (2013).

[41] G. Buchalla, O. Catà, and C. Krause, Complete electroweak chiral Lagrangian with a light Higgs at NLO, Nucl. Phys. B880, 552 (2014); Erratum, Nucl. Phys. B913, 475 (2016).

[42] I. Brivio, T. Corbett, O. J. P. Éboli, M. B. Gavela, J. Gonzalez-Fraile, M. C. Gonzalez-Garcia, L. Merlo, and S. Rigolin, Disentangling a dynamical Higgs, J. High Energy Phys. 03 (2014) 024.

[43] I. Brivio, O. J. P. Éboli, M. B. Gavela, M. C. GonzalezGarcia, L. Merlo, and S. Rigolin, Higgs ultraviolet softening, J. High Energy Phys. 12 (2014) 004.

[44] M. B. Gavela, J. Gonzalez-Fraile, M. C. Gonzalez-Garcia, L. Merlo, S. Rigolin, and J. Yepes, $C P$ violation with a dynamical Higgs, J. High Energy Phys. 10 (2014) 044.

[45] B. M. Gavela, E. E. Jenkins, A. V. Manohar, and L. Merlo, Analysis of general power counting rules in effective field theory, Eur. Phys. J. C 76, 485 (2016).

[46] O. J. P. Éboli and M. C. Gonzalez-Garcia, Classifying the bosonic quartic couplings, Phys. Rev. D 93, 093013 (2016).

[47] I. Brivio, J. Gonzalez-Fraile, M. C. Gonzalez-Garcia, and L. Merlo, The complete Heft Lagrangian after the Lhc Run I, Eur. Phys. J. C 76, 416 (2016).

[48] L. Merlo, S. Saa, and M. Sacristán-Barbero, Baryon noninvariant couplings in Higgs effective field theory, Eur. Phys. J. C 77, 185 (2017).

[49] R. Alonso, K. Kanshin, and S. Saa, Renormalization group evolution of Higgs effective field theory, Phys. Rev. D 97, 035010 (2018).

[50] I. Brivio, M. B. Gavela, S. Pascoli, R. del Rey, and S. Saa, The axion and the Goldstone Higgs, Chin. J. Phys. 61, 55 (2019).

[51] L. Merlo, F. Pobbe, and S. Rigolin, The minimal axion minimal linear $\sigma$ model, Eur. Phys. J. C 78, 415 (2018); Erratum, Eur. Phys. J. C 79, 963 (2019).

[52] J. Alonso-González, L. Merlo, F. Pobbe, S. Rigolin, and O. Sumensari, Testable axion-like particles in the minimal linear $\sigma$ model, Nucl. Phys. B950, 114839 (2020).

[53] J. A. Aguilar-Saavedra, Identifying top partners at Lhc, J. High Energy Phys. 11 (2009) 030.

[54] J. A. Aguilar-Saavedra, R. Benbrik, S. Heinemeyer, and M. Pérez-Victoria, Handbook of vectorlike quarks: Mixing and single production, Phys. Rev. D 88, 094010 (2013).

[55] H.-C. Cheng, I. Low, and L.-T. Wang, Top partners in little Higgs theories with T-parity, Phys. Rev. D 74, 055001 (2006).

[56] S. Banerjee, D. Barducci, G. Bélanger, and C. Delaunay, Implications of a high-mass diphoton resonance for heavy quark searches, J. High Energy Phys. 11 (2016) 154.

[57] B. A. Dobrescu and F. Yu, Exotic signals of vectorlike quarks, J. Phys. G 45, 08LT01 (2018).
[58] M. Chala, Direct bounds on heavy toplike quarks with standard and exotic decays, Phys. Rev. D 96, 015028 (2017).

[59] N. Bizot, G. Cacciapaglia, and T. Flacke, Common exotic decays of top partners, J. High Energy Phys. 06 (2018) 065.

[60] R. Benbrik et al., Signatures of vector-like top partners decaying into new neutral scalar or pseudoscalar bosons, arXiv:1907.05929.

[61] A. De Simone, O. Matsedonskyi, R. Rattazzi, and A. Wulzer, A first top partner Hunter's guide, J. High Energy Phys. 04 (2013) 004.

[62] J. A. Aguilar-Saavedra, D. E. López-Fogliani, and C. Muñoz, Novel signatures for vector-like quarks, J. High Energy Phys. 06 (2017) 095.

[63] S. Colucci, B. Fuks, F. Giacchino, L. L. Honorez, M. H. G. Tytgat, and J. Vandecasteele, Top-philic vectorlike portal to scalar dark matter, Phys. Rev. D 98, 035002 (2018).

[64] G. D’Ambrosio, G. F. Giudice, G. Isidori, and A. Strumia, Minimal flavor violation: An effective field theory approach, Nucl. Phys. B645, 155 (2002).

[65] V. Cirigliano, B. Grinstein, G. Isidori, and M. B. Wise, Minimal flavor violation in the lepton sector, Nucl. Phys. B728, 121 (2005).

[66] S. Davidson and F. Palorini, Various definitions of minimal flavour violation for leptons, Phys. Lett. B 642 , 72 (2006).

[67] R. Alonso, G. Isidori, L. Merlo, L. A. Munoz, and E. Nardi, Minimal flavour violation extensions of the seesaw, J. High Energy Phys. 06 (2011) 037.

[68] D. N. Dinh, L. Merlo, S. T. Petcov, and R. Vega-Álvarez, Revisiting minimal lepton flavour violation in the light of leptonic $C P$ violation, J. High Energy Phys. 07 (2017) 089.

[69] G. Aad et al. (ATLAS Collaboration), Combined measurements of Higgs boson production and decay using up to $80 \mathrm{fb}^{-1}$ of proton-proton collision data at $\sqrt{s}=13 \mathrm{TeV}$ collected with the ATLAS experiment, Phys. Rev. D 101, 012002 (2020)..

[70] M. Aaboud et al. (ATLAS Collaboration), Combination of searches for heavy resonances decaying into bosonic and leptonic final states using $36 \mathrm{fb}^{-1}$ of proton-proton collision data at $\sqrt{s}=13 \mathrm{TeV}$ with the ATLAS detector, Phys. Rev. D 98, 052008 (2018).

[71] P. Bamert, C. P. Burgess, J. M. Cline, D. London, and E. Nardi, $\mathrm{R}(b)$ and new physics: A comprehensive analysis, Phys. Rev. D 54 (1996) 4275.

[72] S. Schael et al. (ALEPH, DELPHI, L3, OPAL, SLD, LEP Electroweak Working Group, SLD Electroweak Group, and SLD Heavy Flavour Group Collaborations), Precision electroweak measurements on the $Z$ resonance, Phys. Rep. 427, 257 (2006).

[73] C. Anastasiou, E. Furlan, and J. Santiago, Realistic composite Higgs models, Phys. Rev. D 79, 075003 (2009).

[74] M. Carena, E. Ponton, J. Santiago, and C. E. M. Wagner, Light Kaluza Klein states in Randall-Sundrum Models with custodial SU(2), Nucl. Phys. B759, 202 (2006). 
[75] J. A. Aguilar-Saavedra, Mixing with vector-like quarks: Constraints and expectations, EPJ Web Conf. 60, 16012 (2013).

[76] J. Alwall, R. Frederix, S. Frixione, V. Hirschi, F. Maltoni, O. Mattelaer, H. S. Shao, T. Stelzer, P. Torrielli, and M. Zaro, The automated computation of tree-level and next-toleading order differential cross sections, and their matching to parton shower simulations, J. High Energy Phys. 07 (2014) 079.

[77] A. Alloul, N. D. Christensen, C. Degrande, C. Duhr, and B. Fuks, FeynRules 2.0 - A complete toolbox for tree-level phenomenology, Comput. Phys. Commun. 185, 2250 (2014).
[78] E. Conte, B. Fuks, and G. Serret, Madanalysis 5, a userfriendly framework for collider phenomenology, Comput. Phys. Commun. 184, 222 (2013).

[79] V. A. Novikov, L. B. Okun, and M. I. Vysotsky, On the electroweak one loop corrections, Nucl. Phys. B397, 35 (1993).

[80] A. Orgogozo and S. Rychkov, The S parameter for a light composite Higgs: A dispersion relation approach, J. High Energy Phys. 06 (2013) 014.

[81] V. Novikov, L. Okun, A. N. Rozanov, and M. Vysotsky, Leptop, arXiv:hep-ph/9503308. 